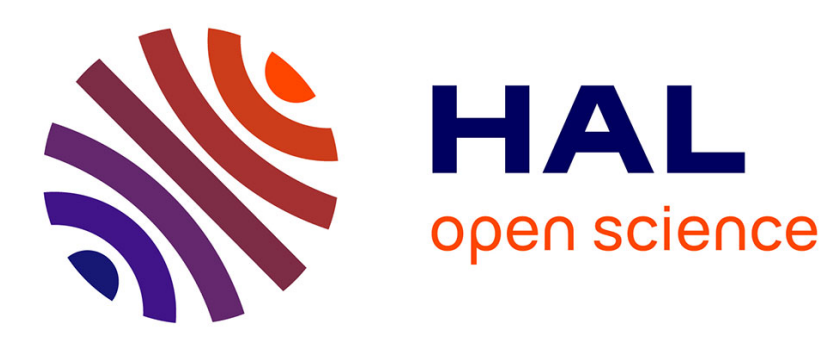

\title{
A compressible multifluid system with new physical relaxation terms
}

Didier Bresch, Matthieu Hillairet

\section{To cite this version:}

Didier Bresch, Matthieu Hillairet. A compressible multifluid system with new physical relaxation terms. Annales Scientifiques de l'École Normale Supérieure, 2019, 52 (2), pp.255-295. 10.24033/asens.2387 . hal-01262617

\section{HAL Id: hal-01262617 https://hal.science/hal-01262617}

Submitted on 27 Jan 2016

HAL is a multi-disciplinary open access archive for the deposit and dissemination of scientific research documents, whether they are published or not. The documents may come from teaching and research institutions in France or abroad, or from public or private research centers.
L'archive ouverte pluridisciplinaire $\mathbf{H A L}$, est destinée au dépôt et à la diffusion de documents scientifiques de niveau recherche, publiés ou non, émanant des établissements d'enseignement et de recherche français ou étrangers, des laboratoires publics ou privés. 


\title{
A COMPRESSIBLE MULTIFLUID SYSTEM WITH NEW PHYSICAL RELAXATION TERMS
}

\author{
D. BRESCH, M. HILLAIRET
}

\begin{abstract}
In this paper, we rigorously derive a new compressible multifluid system from compressible Navier-Stokes equations with density-dependent viscosity in the onedimensional in space setting. More precisely, we propose and mathematically derive a generalization of the usual one velocity Baer-Nunziato model with a new relaxation term in the PDE governing the volume fractions. This new relaxation term encodes the change of viscosity and pressure between the different fluids. For the reader's convenience, we first establish a formal derivation in the bifluid setting using a WKB decomposition and then we rigorously justify the multifluid homogenized system using a kinetic formulation via Young measures characterization.
\end{abstract}

Keywords. Compressible Navier-Stokes, density-dependent viscosity, multifluid systems, Baer-Nunziato, homogenization, Young measures, effective flux, relaxation terms.

AMS classifications. 35Q30, 35D30, 54D30, 42B37, 35Q86, $92 \mathrm{~B} 05$.

\section{INTRODUCTION}

This article is devoted to the mathematical derivation of multifluid systems with one velocity and with variable viscosity. This generalizes the usual Baer-Nunziato system with one velocity, already justified in [6], by modifying the PDE on the fractions through the relaxation term. This term takes into account the change of viscosity and pressure between the different fluids.

If we look at physical books such as those written by M. IsHi and T. HiBIKI (see [14]) or by D. Drew and S.L. PAssman (see [8]), we understand well that it is not so easy to choose the averaging process that has to be used to derive appropriate multifluid systems and that formal closure assumptions are all the times made in physicist's articles. How to derive appropriate multifluid systems reflecting interface flux laws? How to recover mathematically a PDE governing the fraction of each component with physical relaxation terms? These issues require a careful study of the interface evolution between the phases. Unifying the equations for all the phases into a single compressible Navier Stokes equation allows to follow the dynamics of these interfaces (with an appropriate notion of solution enabling to control the divergence of the velocity). Multifluid systems are then interpreted as reduced systems satisfied by particular Young measure (namely convex combinations of a finite number of Dirac masses) solutions to the homogenized compressible Navier Stokes

Date: January 27, 2016. 
equation. Proving propagation of the number of Dirac masses in Young measure solutions to this homogenized equation is then the key-point to derive the multifluid system with new relaxation terms. In this paper, we decide to work on the compressible Navier-Stokes equations with density-dependent viscosity in the one-dimensional in space setting. The density-dependent framework enables to consider phases with different viscosities. We first prove that it is possible to define an appropriate sequence of weak solutions on which we can perform the homogenization process. Our result generalizes to compressible Navier-Stokes equations with density-dependent viscosity the work performed in the one dimension in space by [18] related to compressible Navier-Stokes equations with constant viscosity.

Let describe in more details the method we apply here. Consider for instance in a threedimensional container $\Omega$, the mixture of two viscous compressible phases described by triplets density/velocity/pressure $\left(\rho_{+}, u_{+}, p_{+}\right)$and $\left(\rho_{-}, u_{-}, p_{-}\right)$respectively. Introducing $\left(\mu_{ \pm}, \lambda_{ \pm}\right)$and $\mathcal{P}_{ \pm}$the respective viscosities and pressure laws of the phases, we obtain that, for $i=+,-$ the triplet is a solution to the compressible Navier Stokes equations

$$
\begin{aligned}
\partial_{t} \rho_{i}+\operatorname{div}\left(\rho_{i} u_{i}\right) & =0 \\
\partial_{t}\left(\rho_{i} u_{i}\right)+\operatorname{div}\left(\rho_{i} u_{i} \otimes u_{i}\right) & =\operatorname{div} \Sigma_{i}
\end{aligned}
$$

on its domain $\mathcal{F}_{i}(t)$, with the equation of state:

$$
\begin{aligned}
\Sigma_{i} & =\mu_{i}\left(\nabla u_{i}+\nabla^{\top} u_{i}\right)+\left(\lambda_{i} \operatorname{div} u_{i}-p_{i}\right) \mathbb{I}_{3} \\
p_{i} & =\mathcal{P}_{i}\left(\rho_{i}\right) .
\end{aligned}
$$

Neglecting the properties of the interfaces, so that:

- $\mathcal{F}_{+} \cup \mathcal{F}_{-} \cup\left(\overline{\mathcal{F}_{+}} \cap \overline{\mathcal{F}_{-}}\right)=\Omega$,

- the phases do not slip one on the other at the interface,

- we have continuity of the normal stresses at the interface,

we have that the extended unknowns

$$
\rho=\rho_{+} \mathbf{1}_{\mathcal{F}_{+}}+\rho_{-} \mathbf{1}_{\mathcal{F}_{-}} \quad u=u_{+} \mathbf{1}_{\mathcal{F}_{+}}+u_{-} \mathbf{1}_{\mathcal{F}_{-}}
$$

satisfy the compressible Navier Stokes equations on the whole container $\Omega$ :

$$
\begin{aligned}
\partial_{t} \rho+\operatorname{div}(\rho u) & =0 \\
\partial_{t}(\rho u)+\operatorname{div}(\rho u \otimes u) & =\operatorname{div} \Sigma .
\end{aligned}
$$

Assuming further that the densities of the different phases range two non-overlapping intervals $I_{+}$and $I_{-}$, we can complement this system by the equations of states:

$$
\begin{aligned}
\Sigma_{i} & =2 m\left(\nabla u+\nabla^{\top} u\right)+(l \operatorname{div} u-p) \mathbb{I}_{3} \\
p & =\mathcal{P}(\rho) \quad m=\mathcal{M}(\rho) \quad l=\Lambda(\rho),
\end{aligned}
$$

with functions $\mathcal{P}, \mathcal{M}, \Lambda$ such that for $i \in\{+,-\}$ we have:

$$
\mathcal{P}(\rho)=p_{i}(\rho), \quad \mathcal{M}(\rho)=m_{i}, \quad \Lambda(\rho)=\lambda_{i}, \quad \forall \rho \in I_{i} .
$$

We aim here to compute solutions to this system where any time/space cell of arbitrary small size contains a fraction of phase + and a fraction of phase - . Thus, our problem 
reduces to a mathematical study of the homogenization of solutions to the extended compressible Navier Stokes equation (1)-(4) with respect to initial density. This method for the justification of multifluid systems has been successfully applied in the multi-dimensional setting recently by the authors starting from the compressible Navier-Stokes equation with constant viscosity [6]. The interested reader is also referred to [17, Section 7] where the kinetic equation formulation has been proposed in terms of the cumulative distribution function and without characterization of the Young measures which gives multifluid systems. One corollary of the results in this paper is that the method is robust as it extends to the viscosity-dependent case making precise, in the multifluid setting, previous results initiated by A.A. Amosov and A.A. Zlotnik in the 90's, see [1] and references cited therein. Note also that we do not use the Lagrangian formulation but directly work on the Eulerian system.

In the one-space dimension setting, that we consider in this article, we construct appropriate solutions $(\rho, u, p)$ of the following system

$$
\begin{aligned}
\partial_{t} \rho+\partial_{x}(\rho u) & =0, \\
\partial_{t} \rho u+\partial_{x}\left(\rho u^{2}\right) & =\partial_{x}\left(\mu(\rho) \partial_{x} u\right)-\partial_{x} p, \\
p & =p(\rho)
\end{aligned}
$$

and derive a kinetic equation which governs the evolution of the homogenized system (in terms of Young measures/velocity). With Young measures written as the convex combination of $k$ Dirac masses, we obtain then the multifluid system that reads:

$$
\begin{aligned}
& \partial_{t} \alpha_{i}+\partial_{x}\left(\alpha_{i} u\right)=\frac{\alpha_{i}}{\mu\left(\rho_{i}\right)} f_{i} \\
& \partial_{t} \rho_{i}+u \partial_{x} \rho_{i}=-\frac{\rho_{i}}{\mu\left(\rho_{i}\right)} f_{i} \\
& \partial_{t}(\rho u)+\partial_{x}\left(\rho u^{2}\right)=\partial_{x}\left[\mu \partial_{x} u-p\right]
\end{aligned}
$$

for $1 \leq i \leq k$ with

$$
\begin{aligned}
f_{i} & =\frac{1}{\left[\sum_{j=1}^{k} \frac{\alpha_{j}}{\mu\left(\rho_{j}\right)}\right]}\left(\partial_{x} u-\sum_{j=1}^{k} \alpha_{j} \frac{p\left(\rho_{j}\right)}{\mu\left(\rho_{j}\right)}\right)+p\left(\rho_{i}\right) \quad \text { for } 1 \leq i \leq k \\
\mu & =\frac{1}{\sum_{i=1}^{k} \frac{\alpha_{i}}{\mu\left(\rho_{i}\right)}}, \quad p=\frac{\sum_{i=1}^{k} \frac{\alpha_{i} p\left(\rho_{i}\right)}{\mu\left(\rho_{i}\right)}}{\sum_{i=1}^{k} \frac{\alpha_{i}}{\mu\left(\rho_{i}\right)}} .
\end{aligned}
$$


Note that in the bifluid setting, to get such a system we can also formally search for two-scale solutions (a kind of WKB expansion) under the following form:

$$
\begin{aligned}
& \rho(t, x)=\sum_{i=+,-} \theta_{i}\left(t, \frac{t}{\varepsilon}, x, \frac{x}{\varepsilon}\right) \rho_{i}^{\varepsilon}(t, x), \\
& u(t, x)=u_{0}\left(t, \frac{t}{\varepsilon}, x, \frac{x}{\varepsilon}\right)+\varepsilon u_{1}\left(t, \frac{t}{\varepsilon}, x, \frac{x}{\varepsilon}\right)+\varepsilon^{2} u_{2}\left(t, \frac{t}{\varepsilon}, x, \frac{x}{\varepsilon}\right)+O\left(\varepsilon^{3}\right),
\end{aligned}
$$

assuming that

$$
\rho_{i}^{\varepsilon}(t, x)=\rho_{i}^{0}(t, x)+O(\varepsilon), \quad \theta_{i}(t, \tau, x, y) \in\{0,1\} \quad \text { a.e.. }
$$

After some calculations, the general system that we obtain on $\left(\alpha_{ \pm}, u_{0}, \rho_{ \pm}^{0}, \bar{p}\right)$, where $\alpha_{ \pm}$ denotes the average with respect to the fast variables $(\tau, y)$ of $\theta_{ \pm}$, reads

$$
\begin{aligned}
& \alpha_{+}+\alpha_{-}=1 \\
& \partial_{t} \alpha_{+}+u_{0} \partial_{x} \alpha_{+}=\frac{\alpha_{+} \alpha_{-}}{\alpha_{-} \mu_{+}^{0}+\alpha_{+} \mu_{-}^{0}}\left[\left(p_{+}^{0}-p_{-}^{0}\right)+\left(\mu_{-}^{0}-\mu_{+}^{0}\right) \partial_{x} u_{0}\right] \\
& \partial_{t}\left(\alpha_{+} \rho_{+}^{0}\right)+\partial_{x}\left(\alpha_{+} \rho_{+}^{0} u_{0}\right)=0, \\
& \bar{\rho}\left(\partial_{t} u_{0}+u_{0} \partial_{x} u_{0}\right)-\partial_{x}\left(m \partial_{x} u_{0}\right)+\partial_{x} \pi=0, \\
& \mu_{+}^{0}=\mu\left(\rho_{+}^{0}\right), \quad \mu_{-}^{0}=\mu\left(\rho_{-}^{0}\right), \quad m=\frac{\mu_{+}^{0} \mu_{-}^{0}}{\alpha_{+} \mu_{-}^{0}+\alpha_{-} \mu_{+}^{0}}, \quad \quad \quad \quad \quad p_{-}^{0}=p\left(\rho_{-}^{0}\right), \quad \alpha_{+} \rho_{+}^{0}+\alpha_{-} \rho_{-}^{0}, \quad \pi=\frac{\alpha_{+} p_{+}^{0} \mu_{-}^{0}+\alpha_{-} p_{-}^{0} \mu_{+}^{0}}{\alpha_{+} \mu_{-}^{0}+\alpha_{-} \mu_{+}^{0}} \\
& p_{+}^{0}=p\left(\rho_{+}^{0}\right), \quad
\end{aligned}
$$

Remark that, in the two-fluid setting, we obtain a new equation on the fraction $\alpha_{+}$in which the difference on the effective fluxes plays a crucial role. Namely, we obtain

$$
\partial_{t} \alpha_{+}+u_{0} \partial_{x} \alpha_{+}=\frac{\alpha_{+} \alpha_{-}}{\alpha_{-} \mu_{+}^{0}+\alpha_{+} \mu_{-}^{0}}\left[F_{+}-F_{-}\right] .
$$

where $F_{ \pm}=-\mu_{ \pm}^{0} \partial_{x} u_{0}+p_{ \pm}^{0}$ and $p_{ \pm}^{0}$ and $\mu_{ \pm}^{0}$ are defined by (19)-(18). In the particular case $\mu(\rho)=\mu=$ cste , the system reduces to the one-dimensional system that has been formally derived by W.E. in [12] and fully mathematically justified by D. SERRE in [18]. In that case, the PDE on $\alpha_{+}$simplifies as

$$
\partial_{t} \alpha_{+}+u_{0} \partial_{x} \alpha_{+}=\frac{\alpha_{+} \alpha_{-}}{\mu}\left(p_{+}^{0}-p_{-}^{0}\right) .
$$

In the appendix B, we show that the system obtained through the formal WKB method and the system derived using kinetic formulation and characterization of the Young measures are the same.

The outline of the paper is as follows. We start by our mathematical results. Then, we provide a formal derivation for two-fluid flows plugging the WKB ansatz mentioned above in the compressible Navier-Stokes system with density-dependent viscosity and identifying 
the different terms. Finally, we justify this formal calculation to get a multifluid system with $k$ phases using homogenization technics through Young-measures characterization. The control of the divergence of the velocity field is a key-point of our analysis as it enables to follow the dynamics of the interfaces. In an appendix, we recall some wellknown results on the transport equation and as mentioned previously we compare the result obtained formally and the one obtained through Young-measures characterization in the bifluid setting.

\section{Mathematical Results.}

In this section we make precise the assumptions on the equations of state in the system under consideration. Then, we give the first result of existence which will be used in the homogenization process.

We consider the following Navier-Stokes system with density-dependent viscosity:

$$
\left\{\begin{aligned}
\partial_{t} \rho+\partial_{x}(\rho u) & =0 \\
\partial_{t} \rho u+\partial_{x}\left(\rho u^{2}\right) & =\partial_{x}\left[\mu \partial_{x} u\right]-\partial_{x} p,
\end{aligned} \text { on }(0, L)\right.
$$

completed with the equations of state:

$$
p=p(\rho) \quad \mu=\mu(\rho)
$$

where $p$ and $\mu$ are given and sufficiently smooth: we assume throughout the paper that

$$
\begin{array}{llll}
p \in C^{1}([0, \infty)), & \text { with } \quad p^{\prime}(s) \geq 0, & & \forall s \in[0, \infty) . \\
\mu \in C^{1}([0, \infty)), & \text { with } \quad \mu(s) \geq \mu^{0}(1+\sqrt{s}), & & \forall s \in[0, \infty) .
\end{array}
$$

where $\mu^{0}$ is a given strictly positive constant. The assumption on $p$ can be relaxed into:

$$
p \in C^{1}([0, \infty)), \quad \text { with } \quad p^{\prime}(s) \geq 0, \quad \forall s>>1 .
$$

But we compute with the previous one for simplicity. We complement the above pdes with:

- periodic boundary conditions in $x$

- initial conditions:

$$
\rho(0, x)=\rho^{0}(x), \quad u(0, x)=u^{0}(x) .
$$

Conventions for periodic functions. We denote indifferently with $\sharp$ or quotient-set periodic-function spaces. For instance $L^{\infty}(\mathbb{R} / L \mathbb{Z})=L_{\sharp}^{\infty}$. The symbol $L$ is implicit for any sharped notations. For a Banach space $X$ such as $L^{p}$ or $H^{m}$, we endow $X_{\sharp}$ with the norm:

$$
\|u\|_{X_{\sharp}}=\left\|u_{\left.\right|_{(0, L)}}\right\|_{X(0, L)} .
$$

We recall that $X_{\sharp}$ is a Banach space endowed with this norm and that a sequence $u_{n}$ converges toward $u$ in $X_{\sharp}$ for the strong topology (resp. for the weak or the weak-* topology) if and only if $u_{n}$ converges toward $u$ in $X(-M, M)$ for the strong topology (resp. for the weak or the weak-* topology), whatever the value of $M \in L \mathbb{N}^{*}$. In particular, if $u_{n}$ converges toward $u$ in $X$ (endowed with the weak/weak-* topology) then $u_{n}$ converges toward $u$ in $\mathcal{D}^{\prime}(\mathbb{R})$. 
Our first target result is the following theorem:

Theorem 1. Given $\rho^{0} \in L_{\sharp}^{\infty}$ and $u^{0} \in H_{\sharp}^{1}$ satisfying

$$
\underline{\rho^{0}}:=\inf \rho^{0}(x)>0, \quad \overline{\rho^{0}}=\sup \rho^{0}(x)<\infty,
$$

there exists $T_{0}$ depending on $\underline{\rho^{0}}, \overline{\rho^{0}},\left\|u^{0}\right\|_{H_{\sharp}^{1}}$ such that there exists at least one pair $(\rho, u)$ for which:

$(\mathbf{H D S})_{a}$ we have the regularity statements:

$$
\begin{aligned}
& \rho \in L^{\infty}\left(\left(0, T_{0}\right) ; L_{\sharp}^{\infty}\right) \cap C\left(\left[0, T_{0}\right] ; L_{\sharp}^{1}\right), \\
& u \in L^{\infty}\left(\left(0, T_{0}\right) ; H_{\sharp}^{1}\right) \cap C\left(\left[0, T_{0}\right] ; L_{\sharp}^{2}\right), \\
& z:=\mu(\rho) \partial_{x} u-p(\rho) \in L^{2}\left(\left(0, T_{0}\right) ; H_{\sharp}^{1}\right) ;
\end{aligned}
$$

$(\mathbf{H D S})_{b}(\rho, u)$ satisfies $(20)$ in $\mathcal{D}^{\prime}((0, T) \times \mathbb{R})$, with $p, \mu$ given by $(21)$, and matches initial conditions $(25)$ in $L_{\sharp}^{2} \times H_{\sharp}^{1}$,

$(\text { HDS })_{c}$ we have the following bounds:

- for a.e. $(t, x) \in\left(0, T_{0}\right) \times(\mathbb{R} / L \mathbb{Z})$ there holds:

$$
\frac{1}{2} \rho^{0} \leq \rho(t, x) \leq 2 \overline{\rho^{0}},
$$

- for a.e. $t \in\left(0, T_{0}\right)$ there holds (see (60) for the definition of $q$ ):

$$
\int_{0}^{L}\left[\frac{1}{2}|u(t, \cdot)|^{2}+q(\rho(t, \cdot))\right]+\int_{0}^{t} \int_{0}^{L} \mu\left|\partial_{x} u\right|^{2} \leq \int_{0}^{L}\left[\frac{1}{2}\left|u^{0}\right|^{2}+q\left(\rho^{0}\right)\right]
$$

- there exists a constant $K_{0}$ depending only on $\underline{\rho^{0}}, \overline{\rho^{0}}$ and $\left\|u^{0}\right\|_{H_{\sharp}^{1}}$ for which

$$
\sup _{t \in\left(0, T_{0}\right)}\|u(t, \cdot)\|_{H_{\sharp}^{1}}^{2}+\int_{0}^{T_{0}}\left\|\partial_{x} z(t, \cdot)\right\|_{L_{\sharp}^{2}}^{2} \leq K_{0} .
$$

We call solutions in the sense of Theorem 1 HD solutions to (20)-(21) (after D. HoFF and B. DesJARDins who constructed independently such solutions for the constant viscosity case). The scheme of our proof follows classical lines but we write details for reader's convenience :

- first, we obtain classical solutions to a regularized version of our system using the $\mathrm{BD}$ entropy procedure. This procedure in one-D is well known since the work in 1968 by Y. KANEL in [11]. In our case, "regularized" only means that we assume the initial data to satisfy further $\rho^{0} \in H_{\sharp}^{1}$;

- second, we prove that the strong solutions are HD-solutions on some time-interval $\left(0, T_{0}\right)$ where $T_{0}$ depends only on $\underline{\rho^{0}}, \overline{\rho^{0}},\left\|u^{0}\right\|_{H_{\sharp}^{1}}$;

- third, we apply a compactness argument showing that a sequence of solutions to the regularized system converges to the solution whose existence is claimed in our theorem. These HD solutions provide us with the solutions on which we justify the homogenized procedure through Young measures. 
Enlarging the range of the compactness argument, we also obtain the main result of this paper, namely, the mathematical justification of a generalization of the Baer-Nunziato with one velocity. More precisely we obtain the following mathematical result

Theorem 2. Let $T_{0}>0$ and $\left(\rho_{n}, u_{n}\right)_{n \in \mathbb{N}}$ be a sequence of solutions to (20)-(21) on $\left(0, T_{0}\right)$, in the sense of Theorem 1, with respective initial data $\rho_{n}^{0} \in L_{\sharp}^{\infty}$ and $u_{n}^{0} \in H_{\sharp}^{1}$. Assume that the sequence of initial data satisfies

- $u_{n}^{0} \rightarrow u^{0}$ in $H_{\sharp}^{1}-w$

- there exists a constant $C_{0}>0$ such that $1 / C_{0} \leq \rho_{n}^{0} \leq C_{0}$ uniformly,

- there exists $\left(\alpha_{i}^{0}, \rho_{i}^{0}\right)_{i=1, \ldots, k} \in\left[L_{\sharp}^{\infty}\right]^{2 k}$ such that $\rho_{n}^{0}$ converges in the sense of Young measure (to be defined below) towards

$$
\nu^{0}=\sum_{i=1}^{k} \alpha_{i}^{0} \delta_{\xi=\rho_{i}^{0}}
$$

Then, up to the extraction of a subsequence, $\left(\rho_{n}, u_{n}\right)$ converges to $\left(\left(\alpha_{i}, \rho_{i}\right)_{i=1, \ldots, k}, u\right)$ (in a sense to be made precise) for which we have:

- the regularity statements:

$$
\begin{aligned}
& \alpha_{i} \in L^{\infty}\left(\left(0, T_{0}\right) ; L_{\sharp}^{\infty}\right) \cap C\left(\left[0, T_{0}\right] ; L_{\sharp}^{1}\right) \text { with } \\
& \alpha_{i} \geq 0, \quad \forall i \in\{1, \ldots, k\}, \quad \sum_{i=1}^{k} \alpha_{i}=1, \quad \text { a.e. } \\
& \rho_{i} \in L^{\infty}\left(\left(0, T_{0}\right) ; L_{\sharp}^{\infty}\right) \cap C\left(\left[0, T_{0}\right] ; L_{\sharp}^{1}\right) \text { with } \\
& \quad C_{0} / 2 \leq \rho_{i} \leq 2 C_{0} \text { a.e. } \\
& u \in L^{\infty}\left(\left(0, T_{0}\right) ; H_{\sharp}^{1}\right) \cap C\left(\left[0, T_{0}\right] ; H_{\sharp}^{1}-w\right) ;
\end{aligned}
$$

- the partial differential system (in the sense of $\mathcal{D}^{\prime}\left(\left(0, T_{0}\right) \times \mathbb{R}\right)$ ):

$$
\begin{aligned}
& \partial_{t} \alpha_{i}+\partial_{x}\left(\alpha_{i} u\right)=\frac{\alpha_{i}}{\mu\left(\rho_{i}\right)} f_{i}, \\
& \partial_{t} \rho_{i}+u \partial_{x} \rho_{i}=-\frac{\rho_{i}}{\mu\left(\rho_{i}\right)} f_{i}, \\
& \partial_{t}(\rho u)+\partial_{x}\left(\rho u^{2}\right)=\partial_{x}\left[m \partial_{x} u-\pi\right],
\end{aligned}
$$

where:

$$
\rho=\sum_{j=1}^{k} \alpha_{j} \rho_{j}, \quad m=\left[\sum_{j=1}^{k} \frac{\alpha_{j}}{\mu\left(\rho_{j}\right)}\right]^{-1}, \quad \pi=m \sum_{j=1}^{k} \alpha_{j} \frac{p\left(\rho_{j}\right)}{\mu\left(\rho_{j}\right)} .
$$

and

$$
f_{i}=\frac{1}{\left[\sum_{j=1}^{k} \frac{\alpha_{j}}{\mu\left(\rho_{j}\right)}\right]}\left(\partial_{x} u-\sum_{j=1}^{k} \alpha_{j} \frac{p\left(\rho_{j}\right)}{\mu\left(\rho_{j}\right)}\right)+p\left(\rho_{i}\right)
$$


- the initial conditions:

$$
\begin{aligned}
& \alpha_{i}(0, \cdot)=\alpha_{i}^{0} \text { in } L_{\sharp}^{1}, \\
& \rho_{i}(0, \cdot)=\rho_{i}^{0} \text { in } L_{\sharp}^{1}, \\
& u(0, \cdot)=u^{0} \text { in } H_{\sharp}^{1} .
\end{aligned}
$$

\section{Formal DERIVATION FOR BIFLUID FLOWS.}

In this part, we prove how to get the bifluid system using a formal WKB decomposition. The reader interested by some formal papers related to heat-conducting case or to nonmonotone pressure discussions are referred to [12] and [19].

We assume throughout this section that $(\rho, u)$ is a solution to the compressible Navier Stokes system (5)-(6)-(7) given by the expansion (11)-(12) in which (13) is satisfied.

3.1. General setting. Let us formally multiply the continuity equation by a function $\beta^{\prime}$ such that:

$$
\beta=1 \text { on the support of } \rho_{+}^{0}, \quad \beta=0 \text { on the support of } \rho_{-}^{0} .
$$

We get the classical equation

$$
\partial_{t} \beta(\rho)+\partial_{x}(\beta(\rho) u)+\left(\rho \beta^{\prime}(\rho)-\beta(\rho)\right) \partial_{x} u=0 .
$$

Replacing $\beta(\rho)$ by its value, we get the supplementary equation

$$
\partial_{t} \theta_{+}+u \partial_{x} \theta_{+}=0
$$

Then, we can decompose the derivatives in terms of the slow variables $(t, x)$ and fast variables $(\tau, y)$ of $\theta_{+}$. We get two equations when we consider terms which are $O(1 / \varepsilon)$ or terms which are $O(1)$ :

$$
\begin{aligned}
\partial_{\tau} \theta_{+}+u_{0} \partial_{y} \theta_{+} & =0 \\
\partial_{t} \theta_{+}+u \partial_{x} \theta_{+} & =-\frac{\left(u-u_{0}\right)}{\varepsilon} \partial_{y} \theta_{+} .
\end{aligned}
$$

The first equation provides the behavior of $\theta_{+}$on a cell. This equation is consistent with the assumption that $\theta_{+}$is an indicator function. Averaging with respect to the fast variable the second equation, we get the following PDE on the averaged quantity $\alpha_{+}=\overline{\theta_{+}}$

$$
\partial_{t} \alpha_{+}+\overline{u \partial_{x} \theta_{+}}=-\overline{\frac{\left(u-u_{0}\right)}{\varepsilon} \partial_{y} \theta_{+}} .
$$

We denote temporarily with bars averages on a cell. As this lightens notations a lot, we keep this convention throughout this section only. However, it must not be confused with lower and upper bounds for densities as it has been used in the statement of our Theorem 1 and as will be in the next section. Remark that there is no vacuum in the mixture so that $\theta_{+}+\theta_{-}=1$ a.e.. Consequently, we have:

$$
\alpha_{+}+\alpha_{-}=1 .
$$


Choosing then $\beta^{\prime}$ such that:

$$
\beta=\rho \text { on the support of } \rho_{+}^{0}, \quad \beta=0 \text { on the support of } \rho_{-}^{0} .
$$

We obtain that

$$
\partial_{t}\left(\theta_{+} \rho_{+}^{0}\right)+\partial_{x}\left(\theta_{+} \rho_{+}^{0} u\right)=0
$$

Keeping only the first order in $u$ and averaging with respect to fast variables, we obtain then:

$$
\partial_{t}\left(\alpha_{+} \rho_{+}^{0}\right)+\partial_{x}\left(\rho_{+}^{0} \overline{\theta_{+} u_{0}}\right)=0
$$

Now the main objective is to calculate the averaged terms in (45)-(46) and obtain the momentum equation. To proceed, we use the other equation. We distinguish two cases: the constant viscosity case which gives at the end the system which has been justified recently in [18] (generalized to the multi-dimensioncal case in [6]) and the density-dependent viscosity case which gives at the end the homogenized system under consideration in this paper.

3.2. The constant viscosity case. Plugging the expansion of $u$ in (45), we obtain at first order in $\varepsilon$ :

$$
\partial_{t} \alpha_{+}+\overline{u_{0} \partial_{x} \theta_{+}}=-\overline{u_{1} \partial_{y} \theta_{+}} .
$$

It remains then to compute the averaged quantity on the right-hand side and to justify the homogenized momentum equation. Let us recall quickly the different steps to get the limit system which asks to interprete the divergent parts (in $\varepsilon$ ) of the momentum equation. Indeed, we get the following cascade of equations:

At order $\varepsilon^{-2}$, we get:

$$
\partial_{y y} u_{0}=0 .
$$

The velocity field $u_{0}$ is therefore independent of the fast variable $y$.

At order $\varepsilon^{-1}$, we get then:

$$
\rho^{0}\left(\partial_{\tau} u_{0}+u_{0} \partial_{y} u_{0}\right)=2 \mu \partial_{x y} u_{0}+\mu \partial_{y y} u_{1}-\partial_{y} p^{0} .
$$

(we denote $\rho^{0}=\theta_{+} \rho_{+}^{0}+\theta_{-} \rho_{-}^{0}$ and for the pressure: $p^{0}=\theta_{+} p_{+}^{0}+\theta_{-} p_{-}^{0}$ ). As $u_{0}$ does not depend on $y$ (and $\rho^{0}$ remains far from 0 ), multiplying this equation by $\partial_{\tau} u_{0}$ and integrating on a cell, we obtain $\partial_{\tau} u_{0}=0$. Therefore $u_{0}$ does not depend on both fast variables. In particular

$$
\overline{u_{0} \partial_{x} \theta_{+}}=u_{0} \partial_{x} \alpha_{+}, \quad \overline{\theta_{+} u_{0}}=\alpha_{+} u_{0},
$$

and (46) rewrites:

$$
\partial_{t}\left(\alpha_{+} \rho_{+}^{0}\right)+\partial_{x}\left(\alpha_{+} \rho_{+}^{0} u_{0}\right)=0 .
$$

Using then that $u_{0}$ does not depend on the fast variables in (48) gives, because $\mu$ is constant:

$$
\mu \partial_{y y} u_{1}-\partial_{y} p^{0}=0, \quad \text { and then }, \quad \mu \partial_{y} u_{1}=p^{0}-\overline{p^{0}} .
$$


Multiplying this identity by $\theta_{+}$and taking the average (we recall that $p_{+}^{0}=p\left(\rho_{+}^{0}\right.$ ) and $p_{-}^{0}=p\left(\rho_{-}^{0}\right)$ do not dependent of the fast variable), we get

$$
-\overline{u_{1} \partial_{y} \theta_{+}}=\overline{\theta_{+} \partial_{y} u_{1}}=\frac{1}{\mu} \overline{\theta_{+}\left(p^{0}-\overline{p^{0}}\right)}=\frac{\alpha_{+} \alpha_{-}}{\mu}\left(p_{+}^{0}-p_{-}^{0}\right) .
$$

Finally, we obtain the expected equation for the volume fraction:

$$
\partial_{t} \alpha_{+}+u_{0} \partial_{x} \theta_{+}=\frac{\alpha_{+} \alpha_{-}}{\mu}\left(p_{+}^{0}-p_{-}^{0}\right) .
$$

At order $\varepsilon^{0}$, in the momentum equation, we have now:

$$
\rho^{0} \partial_{t} u_{0}+\rho^{0} u_{0} \partial_{x} u_{0}+\rho^{0}\left(\partial_{\tau} u_{1}+u_{0} \partial_{y} u_{1}\right)=\partial_{x} \Sigma_{0}+\partial_{y} \Sigma_{1}
$$

where

$$
\Sigma_{0}=\mu\left(\partial_{x} u_{0}+\partial_{y} u_{1}\right)-\sum_{i=+,-} \theta_{i} p\left(\rho_{i}^{0}\right) .
$$

On the left-hand side, we recall that

$$
\partial_{y} u_{1}=\frac{1}{\mu}\left(\sum_{i=+,-} \theta_{i} p_{i}^{0}-\bar{p}^{0}\right)
$$

and, in terms of the fast variables $(\tau, y), \partial_{y} u_{1}$ is thus a linear function of $\theta_{ \pm}$only so that (43) induces that

$$
\partial_{y}\left(\partial_{\tau} u_{1}+u_{0} \partial_{y} u_{1}\right)=0
$$

and consequently (because $\partial_{\tau} u_{1}+u_{0} \partial_{y} u_{1}$ has average 0 on a cell):

$$
\partial_{\tau} u_{1}+u_{0} \partial_{y} u_{1}=0 \text {. }
$$

Taking the average of (51) w.r.t fast variables, we obtain finally:

$$
\bar{\rho} \partial_{t} u_{0}+\bar{\rho} u_{0} \partial_{x} u_{0}=\partial_{x} \bar{\Sigma}_{0}
$$

with

$$
\bar{\rho}=\alpha_{+} \rho_{+}^{0}+\alpha_{-} \rho_{-}^{0}, \quad \bar{\Sigma}_{0}=\mu \partial_{x} u_{0}-\sum_{i=+,-} \alpha_{i} p_{i}^{0} .
$$

Combining with (49)-(50), this completes the justification of the bifluid system in the constant-viscosity case.

3.3. The density-dependent viscosity case. In this second case, we go back to the relation

$$
\partial_{t} \theta_{+}+u_{0} \partial_{x} \theta_{+}=-u_{1} \partial_{y} \theta_{+}
$$

that we want to average. We write again the different scales on the momentum equations. We recall that we assume density-dependent viscosity $\mu=\mu(\rho)$. Therefore we can write

$$
\mu=\theta_{+} \mu_{+}^{\varepsilon}+\theta_{-} \mu_{-}^{\varepsilon}
$$

where we assume at first order that $\mu_{ \pm}^{\varepsilon} \sim \mu_{ \pm}^{0}$ which does not depend on the fast variables. 
Order $\varepsilon^{-2}$. We get

$$
\partial_{y}\left[\mu \partial_{y} u_{0}\right]=0
$$

This implies that

$$
\mu \partial_{y} u_{0}=K_{0} .
$$

To determine $K_{0}$ we use the equation at order $\varepsilon^{-1}$ for $\theta_{+}$(and $\theta_{-}$) (43) that we multiply by $\mu_{+}^{\varepsilon}$ (et $\mu_{-}^{\varepsilon}$ respectively). After some combinations, we get:

$$
\partial_{\tau} \mu+u_{0} \partial_{y} \mu=0
$$

Averaging the equation on a cell, we get that

$$
0=\overline{u_{0} \partial_{y} \mu}=-\overline{\mu \partial_{y} u_{0}}=K_{0}
$$

Finally, we get that $\partial_{y} u_{0}=0$ and therefore $u_{0}$ does not depend of the space fast variables.

Order $\varepsilon^{-1}$. We get with the same arguments as previously

$$
\rho^{0} \partial_{\tau} u_{0}=\partial_{y}\left[\mu^{0}\left(\partial_{y} u_{1}+\partial_{x} u_{0}\right)\right]-\partial_{y} p^{0}
$$

and therefore, because $\partial_{\tau} u_{0}$ is constant, we obtain $\partial_{\tau} u_{0}=0$ after multiplication by $\partial_{\tau} u_{0}$ and integration on a cell. Hence, $u_{0}$ does not depend on both fast variables again and we obtain (49). Also, the above equation then reduces to:

$$
0=\partial_{y}\left[\mu^{0}\left(\partial_{y} u_{1}+\partial_{x} u_{0}\right)\right]-\partial_{y} p^{0} .
$$

This gives

$$
\mu^{0} \partial_{y} u_{1}+\left(\mu^{0}-\overline{\mu^{0}}\right) \partial_{x} u_{0}-\left(p^{0}-\bar{p}^{0}\right)=K_{1}
$$

Let us note that at first order $K_{1}=\overline{\mu^{0} \partial_{y} u_{1}}$. We want to calculate this quantity. To calculate $K_{1}$, we proceed as previously, we multiply (52) by $\mu_{+}^{0}$ (and its equivalent for $\theta_{-}$ by $\left.\mu_{-}^{0}\right)$. After some combinations, this gives:

$$
\partial_{t} \mu^{0}+u_{0} \partial_{x} \mu^{0}+u_{1} \partial_{y} \mu^{0}=\sum_{i= \pm} \theta_{i}\left(\partial_{t}+u_{0} \partial_{x}\right) \mu_{i}^{0} .
$$

Averaging with respect to the fast variable, we get

$$
\begin{aligned}
\overline{u_{1} \partial_{y} \mu^{0}} & =\sum_{i= \pm} \alpha_{i}\left(\partial_{t}+u_{0} \partial_{x}\right) \mu_{i}^{0}-\left(\partial_{t}+u_{0} \partial_{x}\right) \overline{\mu^{0}} \\
& =-\sum_{i= \pm} \mu_{i}^{0}\left(\partial_{t}+u_{0} \partial_{x}\right) \alpha_{i} \\
& =\left(\mu_{-}^{0}-\mu_{+}^{0}\right)\left(\partial_{t}+u_{0} \partial_{x}\right) \alpha_{+}
\end{aligned}
$$

and finally

$$
K_{1}=\overline{\mu^{0} \partial_{y} u_{1}}=\left(\mu_{+}^{0}-\mu_{-}^{0}\right)\left(\partial_{t}+u_{0} \partial_{x}\right) \alpha_{+}
$$


Thus we can calculate at first order:

$$
\begin{aligned}
-\overline{u_{1} \partial_{y} \theta_{+}} & =\overline{\theta_{+} \partial_{y} u_{1}} \\
& =\frac{\theta_{+}}{\mu^{0}}\left(p^{0}-\overline{p^{0}}\right)-\frac{\theta_{+}}{\mu^{0}}\left(\mu^{0}-\overline{\mu^{0}}\right) \partial_{x} u_{0}+\frac{\theta_{+}}{\mu^{0}} K_{1}
\end{aligned}
$$

Then we have

$$
\begin{aligned}
\overline{\frac{\theta_{+}}{\mu^{0}}\left(p^{0}-\bar{p}^{0}\right)} & =\frac{\alpha_{+}}{\mu_{+}^{0}} p_{+}^{0}-\frac{\alpha_{+}}{\mu_{+}^{0}}\left(\alpha_{+} p_{+}^{0}+\alpha_{-} p_{-}^{0}\right) \\
& =\frac{\alpha_{+} \alpha_{-}}{\mu_{+}^{0}}\left(p_{+}^{0}-p_{-}^{0}\right),
\end{aligned}
$$

and on the other part

$$
\overline{\frac{\theta_{+}}{\mu^{0}} K_{1}}=\frac{\alpha_{+}}{\mu_{+}^{0}}\left(\mu_{+}^{0}-\mu_{-}^{0}\right)\left(\partial_{t}+u_{0} \partial_{x}\right) \alpha_{+}
$$

and finally :

$$
\overline{\frac{\theta_{+}}{\mu^{0}}\left(\mu^{0}-\bar{\mu}^{0}\right) \partial_{x} u_{0}}=\partial_{x} u_{0} \frac{\alpha_{+} \alpha_{-}}{\mu_{+}^{0}}\left(\mu_{+}^{0}-\mu_{-}^{0}\right) .
$$

Thus

$$
\overline{-u_{1} \partial_{y} \theta_{+}}=\frac{\alpha_{+} \alpha_{-}}{\mu_{+}^{0}}\left(\left(p_{+}^{0}-p_{-}^{0}\right)-\partial_{x} u_{0}\left(\mu_{+}^{0}-\mu_{-}^{0}\right)\right)+\alpha_{+}\left(1-\frac{\mu_{-}^{0}}{\mu_{+}^{0}}\right)\left(\partial_{t}+u_{0} \partial_{x}\right) \alpha_{+}
$$

Therefore we get finally the following equation on $\alpha_{+}$:

$$
\left(1+\alpha_{+}\left(\frac{\mu_{-}^{0}}{\mu_{+}^{0}}-1\right)\right)\left(\partial_{t} \alpha_{+}+u_{0} \partial_{x} \alpha_{+}\right)=\frac{\alpha_{+} \alpha_{-}}{\mu_{+}^{0}}\left(\left(p_{+}^{0}-p_{-}^{0}\right)-\partial_{x} u_{0}\left(\mu_{+}^{0}-\mu_{-}^{0}\right)\right)
$$

which may be rewritten as:

$$
\partial_{t} \alpha_{+}+u_{0} \partial_{x} \alpha_{+}=\frac{\alpha_{+} \alpha_{-}}{\alpha_{+} \mu_{-}^{0}+\alpha_{-} \mu_{+}^{0}}\left(\left(p_{+}^{0}-p_{-}^{0}\right)-\partial_{x} u_{0}\left(\mu_{+}^{0}-\mu_{-}^{0}\right)\right) .
$$

As for the momentum equation, we write the $\varepsilon^{0}$ order of the momentum equation as in the previous case. We remark again that, thanks to (53), the quantity $\partial_{y} u_{1}$ depends on the fast variable only through $\theta_{ \pm}$so that after averaging, we obtain:

$$
\bar{\rho} \partial_{t} u_{0}+\bar{\rho} u_{0} \partial_{x} u_{0}=\partial_{x} \bar{\Sigma}_{0}
$$

with

$$
\bar{\rho}=\alpha_{+} \rho_{+}^{0}+\alpha_{-} \rho_{-}^{0}, \quad \bar{\Sigma}_{0}=\mu^{0} \partial_{x} u_{0}-\sum_{i=+,-} \alpha_{i} p_{i}^{0}+\overline{\mu^{0} \partial_{y} u_{1}} .
$$

Combining (54) and (56), we have:

$$
\overline{\mu^{0} \partial_{y} u_{1}}=\frac{\alpha_{+} \alpha_{-}\left(\mu_{+}^{0}-\mu_{-}^{0}\right)}{\alpha_{+} \mu_{-}^{0}+\alpha_{-} \mu_{+}^{0}}\left(\left(p_{+}^{0}-p_{-}^{0}\right)-\partial_{x} u_{0}\left(\mu_{+}^{0}-\mu_{-}^{0}\right)\right),
$$


so that, after tedious but straightfoward algebraic combination (using many times that $\alpha_{+}+\alpha_{-}=1$ ), we get:

$$
\bar{\Sigma}_{0}=\frac{\mu_{+}^{0} \mu_{-}^{0}}{\alpha_{+} \mu_{-}^{0}+\alpha_{-} \mu_{+}^{0}} \partial_{x} u_{0}-\frac{\alpha_{+} p_{+}^{0} \mu_{-}^{0}+\alpha_{-} p_{-}^{0} \mu_{+}^{0}}{\alpha_{+} \mu_{-}^{0}+\alpha_{-} \mu_{+}^{0}} .
$$

This completes the justification of the bifluid system mentioned in the introduction.

\section{Mathematical proofs.}

In this section, we mathematically justify the derivation of a multifluid system with variable viscosities from the compressible Navier-Stokes system with a density-dependent viscosity. We consider the one-dimensional in space case to be able to construct global strong solutions far from vacuum in the classical setting. We therefore recall and make precise the result of existence and method of proof coming from [16] and recently [10]. Then, we perform the compactness result and derive the multifluid system.

4.1. Strong solution theory. By adapting the arguments of [16] to our periodic framework, we have the following existence theorem

Theorem 3. Given $\rho^{0} \in H_{\sharp}^{1}$ and $u^{0} \in H_{\sharp}^{1}$ satisfying

$$
\underline{\rho^{0}}:=\inf \rho^{0}(x)>0,
$$

there exists a unique pair $(\rho, u)$ such that:

$(\mathbf{C S})_{a}$ we have the regularity statement

$$
\begin{aligned}
& \rho \in C\left([0, \infty) ; H_{\sharp}^{1}\right) \text { with } \rho>0, \\
& u \in C\left([0, \infty) ; H_{\sharp}^{1}\right) \cap L_{l o c}^{2}\left((0, \infty) ; H_{\sharp}^{2}\right) ;
\end{aligned}
$$

$(\mathbf{C S})_{b}(\rho, u)$ satisfies $(20)$ a.e. in $(0, \infty) \times \mathbb{R}$ with $p, \mu$ given by $(21)$;

$(\mathbf{C S})_{c}(\rho, u)$ matches initial conditions (25) a.e..

We sketch the proof of this theorem for completeness.

Local existence of solutions is obtained by a classical fixed-point argument so that the only difficulty lies in proving these solutions are global. As the local-in-time theory yields a time of existence depending only on

$$
\mathcal{E}(0):=\underline{\rho^{0}}+\left\|\rho^{0}\right\|_{H_{\sharp}^{1}}+\left\|u^{0}\right\|_{H_{\sharp}^{1}},
$$

we aim to obtain a local-in-time uniform bound on $\mathcal{E}(t)$ for the associated solution $(\rho, u)$. This solution is defined a priori on a non-extendable time interval $\left[0, T_{*}\right)$. 
Step 1. Dissipation of energy. First, with classical arguments, we obtain

$$
\begin{aligned}
\int_{0}^{L}\left[\frac{\rho(t, x)|u(t, x)|^{2}}{2} \mathrm{~d} x+q(\rho(t, x))\right]+\int_{0}^{t} & \int_{0}^{L} \mu(t, x)\left|\partial_{x} u(s, x)\right|^{2} \mathrm{~d} x \mathrm{~d} t \\
& =\int_{0}^{L}\left[\frac{\rho^{0}(x)\left|u^{0}(x)\right|^{2}}{2} \mathrm{~d} x+q\left(\rho^{0}(x)\right)\right] \mathrm{d} x
\end{aligned}
$$

for all $t \in\left[0, T_{*}\right)$ where $q$ is defined by:

$$
q(z)=z \partial_{s}^{-1}\left\{\frac{p(s)}{s^{2}}\right\}
$$

Step 2. BD entropy. We control now the growth of the $H^{1}$-norm of $\rho$. Namely, we adapt to our periodic case the BD-entropy method which may be found in its simplest form in [5] for instance. So, we introduce $\varphi \in C^{1}((0, \infty))$ defined by

$$
\varphi(z)=\int_{1}^{z} \frac{\mu(s)}{s^{2}} \mathrm{~d} s, \quad \forall z \in(0, \infty) .
$$

Note that for a nonlinear function $\varphi_{1}$ of the density, we have

$$
\partial_{t} \varphi_{1}(\rho)+u \partial_{x} \varphi_{1}(\rho)+\varphi_{1}^{\prime}(\rho) \rho \partial_{x} u=0 .
$$

Thus differentiating with respect to space

$$
\partial_{t} \partial_{x}\left(\varphi_{1}(\rho)\right)+\partial_{x}\left(u \partial_{x} \varphi_{1}(\rho)\right)+\partial_{x}\left(\varphi_{1}^{\prime}(\rho) \rho \partial_{x} u\right)=0 .
$$

Let us now choose $\varphi_{1}(\rho)=\int_{1}^{\rho} \mu(\rho) / \rho$, then we get from the definition of $\varphi$

$$
\partial_{t}\left(\rho \partial_{x} \varphi(\rho)\right)+\partial_{x}\left(\rho u \partial_{x} \varphi(\rho)\right)+\partial_{x}\left(\mu(\rho) \partial_{x} u\right)=0 .
$$

Adding the relation to the momentum equation gives

$$
\partial_{t}\left(\rho\left(u+\partial_{x} \varphi(\rho)\right)\right)+\partial_{x}\left(\rho u\left(u+\partial_{x} \varphi(\rho)\right)\right)+\partial_{x} p(\rho)=0 .
$$

In what follows, we denote $\varphi_{x}:=\partial_{x} \varphi(\rho(x))$ to be distinguished with $z \rightarrow \varphi^{\prime}(z)$ the simple derivative of the above defined function $\varphi$. We keep subscript $x$ to denote partial derivatives w.r.t. space variable (we have thus $\partial_{x} u=u_{x}$ ). Testing the equation (61) with $u+\partial_{x} \varphi$ yields finally:

$$
\frac{1}{2} \frac{\mathrm{d}}{\mathrm{d} t}\left[\int_{0}^{L}\left\{\rho\left|u+\varphi_{x}\right|^{2}+q(\rho)\right\}\right]+\int_{0}^{L} p^{\prime} \varphi^{\prime}\left|\rho_{x}\right|^{2}=0 .
$$

As $p^{\prime} \varphi^{\prime} \geq 0$ we conclude that

$$
\int_{0}^{L}\left\{\rho\left|u+\varphi_{x}\right|^{2}+q(\rho)\right\} \leq C_{0}, \quad \forall t \geq 0 .
$$

Hence:

$$
\int_{0}^{L}\left|\sqrt{\rho} \varphi^{\prime}(\rho) \rho_{x}\right|^{2} \leq C_{0}, \quad \forall t \geq 0
$$


As the continuity equation implies the conservation of the mean of $\rho$ on $(0, L)$ we derive that, setting $f \in C^{1}((0, \infty))$ any primitive of $z \mapsto \mu(z) / z^{3 / 2}$, there holds:

$$
\|f(\rho(t, \cdot))\|_{L_{\sharp}^{\infty} \leq C_{0}, \quad \forall t \geq 0 .}
$$

In particular, our assumption (23) on $\mu$ enforces that $f(z)$ diverges when $z \rightarrow 0$ or $z \rightarrow \infty$. Hence, we obtain from the control above that

$$
\|\rho(t, \cdot)\|_{L_{\sharp}^{\infty}}+\left\|\rho^{-1}(t, \cdot)\right\|_{L_{\sharp}^{\infty}} \leq C_{0}, \quad \forall t \geq 0,
$$

and, plugging this inequality into (63) (and applying again that the mean of $\rho$ is constant with time so that the $\left\|\partial_{x} \rho\right\|_{L_{\sharp}^{2}}$ controls the $H^{1}$-norm of $\rho$ ), we get:

$$
\|\rho(t, \cdot)\|_{H_{\sharp}^{1}} \leq C_{0}, \quad \forall t \geq 0 .
$$

From the BD-entropy argument we developed up to now, we obtain global-in-time control on the $\rho$ in the $H^{1}$-norm and in the $L^{\infty}$-norm from above and from below.

\section{Remarks.}

1. In case $p$ merely satisfies (24), equation (62) induces that for a constant $C_{p \mu}>0$ there holds:

$$
\frac{1}{2} \frac{\mathrm{d}}{\mathrm{d} t}\left[\int_{0}^{L}\left\{\rho\left|u+\varphi_{x}\right|^{2}+q(\rho)\right\}\right] \leq C_{p \mu} \int_{0}^{L} \rho\left|\varphi_{x}\right|^{2} .
$$

Hence, recalling that the total energy of the solution remains uniformy bounded with time, we obtain, by applying a Gronwall lemma, that there exists a positive constant $C_{0}$ depending only on initial data, for which:

$$
\int_{0}^{L}\left\{\rho\left|u+\varphi_{x}\right|^{2}+q(\rho)\right\} \leq C_{0} C_{p \mu}(1+t) \exp \left(2 C_{p \mu} t\right) \quad \forall t \geq 0 .
$$

2. Recently, B. HAspot has extended the range of viscosity that provides global existence of strong solution for the compressible Navier-Stokes equation with densitydependent viscosity if initially the density is far from vacuum. His nice idea is to remark that the equation on $v=u+\partial_{x} \varphi(\rho)$ contains a damping term if we replace the pressure term in terms of the $v$ and $u$. More precisely, we get the equation

$$
\partial_{t}(\rho v)+\partial_{x}(\rho u v)+\frac{p^{\prime}(\rho) \rho^{2}}{\mu(\rho)} v=\frac{p^{\prime}(\rho) \rho^{2}}{\mu(\rho)} u .
$$

Thus for $p(s)=a s^{\gamma}(\gamma>1)$ if we assume $\mu(s) \leq C+C p(s)$ for all $s \geq 0$ then he first proves that $v$ is $L^{\infty}\left(0, T ; L^{\infty}\right)$ and then coming back to the mass equation that $1 / \rho$ belongs to $L^{\infty}$. This allows him to extend a local in time result to a global one. In conclusion, our homogenized result may for instance be extended to the shallow-water system where $\mu(\rho)=\rho$ and $p(\rho)=a \rho^{2}$.

Step 3. Regularity. Finally, we obtain propagation of the $H^{1}$ regularity for $u$. Namely, we differentiate once the momentum equation, yielding:

$$
\rho\left(\partial_{t} u_{x}+u \partial_{x} u_{x}\right)=\partial_{x}\left(\mu \partial_{x} u_{x}\right)-p_{x x}-\rho_{x}\left(\partial_{t} u+u \partial_{x} u\right)-\rho\left|u_{x}\right|^{2}+\partial_{x}\left(\mu_{x} u_{x}\right) .
$$


Multiplying this equality with $u_{x}$ yields:

$\frac{1}{2} \frac{\mathrm{d}}{\mathrm{d} t}\left[\int_{0}^{L} \rho\left|u_{x}\right|^{2}\right]+\int_{0}^{L} \mu\left|\partial_{x} u_{x}\right|^{2}=-\int_{0}^{L}\left(p_{x x}+\rho_{x}\left(\partial_{t} u+u \partial_{x} u\right)+\rho\left|u_{x}\right|^{2}\right) u_{x}-\int_{0}^{L} \mu_{x} \partial_{x} u_{x} u_{x}$.

On the right-hand side, we have after integration by parts:

$$
\left|\int_{0}^{L} p_{x x} u_{x}\right| \leq \frac{C}{\mu^{0} \varepsilon}\|p\|_{H^{1}(0, L)}^{2}+\varepsilon \int_{0}^{L} \mu\left|\partial_{x} u_{x}\right|^{2} .
$$

Then, we replace

$$
\rho_{x}\left(\partial_{t} u+u \partial_{x} u\right)=\frac{\rho_{x}}{\rho}\left[\mu u_{x x}+\mu_{x} u_{x}-p_{x}\right]
$$

so that:

$$
\begin{aligned}
\left|\int_{0}^{L} \rho_{x}\left(\partial_{t} u+u \partial_{x} u\right) u_{x}\right| \leq & \|\mu\|_{L_{\sharp}^{\infty}}^{\frac{1}{2}}\left\|\rho^{-1}\right\|_{L_{\sharp}^{\infty}}\|\rho\|_{H_{\sharp}^{1}}\left\|u_{x}\right\|_{L_{\sharp}^{\infty}}\left(\int_{0}^{L} \mu\left|u_{x x}\right|^{2}\right)^{\frac{1}{2}} \\
& +\left\|\rho^{-1}\right\|_{L_{\sharp}^{\infty}}\|\rho\|_{H_{\sharp}^{1}}\left\|\mu_{x}\right\|_{L_{\sharp}^{2}}\left\|u_{x}\right\|_{L_{\sharp}^{\infty}}^{2} \\
& +\left\|\rho^{-1}\right\|_{L_{\sharp}^{\infty}}\|\rho\|_{H_{\sharp}^{1}}\left\|p_{x}\right\|_{L_{\sharp}^{2}}\left\|u_{x}\right\|_{L_{\sharp}^{\infty}} \\
\leq & C_{0}\left(1+\left\|u_{x}\right\|_{L_{\sharp}^{2}}^{2}\right)+\varepsilon \int_{0}^{L} \mu\left|u_{x x}\right|^{2}
\end{aligned}
$$

where we applied the previous controls on $\rho$ in the $H^{1}$ and $L^{\infty}$ norms, and that, for an absolute constant $C$, there holds:

$$
\left\|u_{x}\right\|_{L_{\sharp}^{\infty}} \leq C\left\|u_{x}\right\|_{L_{\sharp}^{2}}^{\frac{1}{2}}\left\|u_{x x}\right\|_{L_{\sharp}^{2}}^{\frac{1}{2}} .
$$

We have similarly:

$$
\left.\left.\left|\int_{0}^{L} \rho\right| u_{x}\right|^{2} u_{x}\left|\leq \frac{C_{0}}{2 \varepsilon}\left(1+\left\|u_{x}\right\|_{L_{\sharp}^{2}}^{4}\right)+\varepsilon \int_{0}^{L} \mu\right| u_{x x}\right|^{2} .
$$

and

$$
\left|\int_{0}^{L} \mu_{x} u_{x} \partial_{x} u_{x}\right| \leq \frac{C_{0}}{\varepsilon}\left\|u_{x}\right\|_{L_{\sharp}^{2}}^{2}+\varepsilon \int_{0}^{L} \mu\left|u_{x x}\right|^{2}
$$

Combining all these computations in our first identity, and choosing $\varepsilon$ sufficiently small, yields:

$$
\frac{1}{2} \frac{\mathrm{d}}{\mathrm{d} t}\left[\int_{0}^{L} \rho\left|u_{x}\right|^{2}\right]+\frac{1}{2} \int_{0}^{L} \mu\left|\partial_{x} u_{x}\right|^{2}=C_{0}\left(1+\left\|u_{x}\right\|_{L_{\sharp}^{2}}^{4}\right)
$$

Applying a standard Gronwall inequality and recalling the dissipation of energy estimate, we obtain then that:

$$
\int_{0}^{L}\left|u_{x}(t, x)\right|^{2} \mathrm{~d} x \leq C_{0}(1+t) \exp \left(C_{0}\right), \quad \forall t \geq 0
$$

This ends the proof. 
4.2. Uniform estimates. Prior to establishing Theorem 1, we show in this section that the global strong solutions of the previous section, that we construct for initial data $\left(\rho^{0}, u^{0}\right) \in H_{\sharp}^{1} \times H_{\sharp}^{1}$, do satisfy the requirements $(\mathrm{HDS})_{a},(\mathrm{HDS})_{b}$ and $(\mathrm{HDS})_{c}$ of Theorem 1 on some time interval $\left(0, T_{0}\right)$ with $T_{0}$ depending only on $\underline{\rho^{0}}, \overline{\rho^{0}},\left\|u^{0}\right\|_{H_{\sharp}^{1}}$. This completes the proof of Theorem 1 in the case $\rho^{0}$ satisfies the further property $\rho^{0} \in H_{\sharp}^{1}$.

So, let $\left(\rho^{0}, u^{0}\right) \in H_{\sharp}^{1} \times H_{\sharp}^{1}$ and $(\rho, u)$ the associated global strong solution given by Theorem 3. Clearly, $(\mathrm{CS})_{a}$ (resp. $\left.(\mathrm{CS})_{b}\right)$ induces that $(\mathrm{HDS})_{a}\left(\operatorname{resp} .(\mathrm{HDS})_{b}\right)$ holds on arbitrary time-interval $\left(0, T_{0}\right)$. We remind also that this solution satisfies the dissipation energy estimate (59). Hence, denoting by

$$
\mathcal{E}_{0}^{c}:=\int_{0}^{L}\left[\frac{\overline{\rho^{0}}\left|u^{0}(x)\right|^{2}}{2} \mathrm{~d} x+\max _{\left[\underline{\rho^{0}}, \overline{\rho^{0}}\right]} q(z)\right] \mathrm{d} x
$$

we have that, for arbitrary $T_{0}>0$ :

$$
\sup _{t \in\left(0, T_{0}\right)}\left[\frac{1}{2} \int_{0}^{L} \rho(t, x)|u(t, x)|^{2} \mathrm{~d} x+\int_{0}^{t} \int_{0}^{L} \mu(s, x)\left|\partial_{x} u(s, x)\right| \mathrm{d} s \mathrm{~d} x\right] \leq \mathcal{E}_{c}^{0} .
$$

The only point is thus to obtain the bounds (30) and (32). Note also that thanks to the regularity $(\mathbf{C S})_{a}$, these conditions are indeed satisfied but for a sufficiently small $\tilde{T}_{0}$ only. The actual difficulty is thus to prove that we may choose $\tilde{T}_{0}=T_{0}$ depending only on $\underline{\rho^{0}}, \overline{\rho^{0}},\left\|u^{0}\right\|_{H_{\sharp}^{1}}$. For this purpose, in what follows, we pick a positive time $\tilde{T}_{0}$ for which (30) and $(32)$ are satisfied by $(\rho, u)$ on $\left[0, \tilde{T}_{0}\right]$ for a well chosen $K^{0}$. We show then, that, if we assume $\tilde{T}_{0}<T_{0}$, for some $T_{0}$ to be constructed with the expected dependencies, we obtain a better bound for $(\rho, u)$. By a standard connectedness argument ${ }^{1}$, we obtain then that we may choose $\tilde{T}_{0}=T_{0}$.

For the computations below, we introduce the following notations:

- we introduce the function $\kappa=p / \mu$

- given $\beta \in C([0, \infty))$, we denote

$$
\begin{gathered}
K_{\beta}^{0}=\max \left\{\beta(z), z \in\left[\underline{\left.\left.\rho^{0} / 2,2 \overline{\rho^{0}}\right]\right\}}\right.\right. \\
\text { - } K_{u}^{0}=36\left(\frac{1}{\mu^{0}}+\overline{\rho^{0}}\right)\left[\left\|\sqrt{\mu\left(\rho^{0}\right)} \partial_{x} u_{0}-\kappa\left(\rho^{0}\right)\right\|_{L_{\sharp}^{2}}^{2}+1+L\left|K_{\kappa}^{0}\right|^{2}\right] .
\end{gathered}
$$

We remark that $K_{u}^{0}$ do depend only on $\overline{\rho^{0}}, \underline{\rho^{0}},\left\|u^{0}\right\|_{H_{\sharp}^{1}}$. It will play the role of $K^{0}$ in our proof.

\footnotetext{
${ }^{1}$ Given the regularity statements $(\mathbf{C S})_{a}$ the following quantities are continuous functions of timevariable $t \in[0, \infty)$ :

$$
\min _{[0, L]} \rho(t, x), \quad \max _{[0, L]} \rho(t, x), \quad \int_{0}^{t}\left\|\partial_{x} z\right\|_{L_{\sharp}^{2}}^{2}, \quad \sup _{(0, t)}\|u\|_{H_{\sharp}^{1}}
$$
}


According to the method of proof we described above, we assume from now on that $\tilde{T}_{0}>0$ is chosen and fixed such that we have the a priori bounds:

$$
\begin{gathered}
\frac{\rho^{0}}{\overline{2}} \leq \rho(t, x) \leq 2 \overline{\rho^{0}} \quad \text { on }\left(0, \tilde{T}_{0}\right) \times \mathbb{R} \\
\sup _{\left(0, \tilde{T}_{0}\right)}\|u\|_{H_{\sharp}^{1}}^{2}+\int_{0}^{\tilde{T}_{0}}\left\|\partial_{x} z\right\|_{L_{\sharp}^{2}}^{2} \mathrm{~d} s \leq K_{u}^{0} .
\end{gathered}
$$

We state first the following lemma:

Lemma 4. Let denote:

$$
K_{d}^{0}=\frac{1}{\mu^{0}}\left(\sqrt{L}+\frac{1}{\sqrt{L}}\right)\left(2 K_{\mu}^{0} \mathcal{E}_{0}^{c}+2 L\left|K_{p}^{0}\right|^{2}+K_{u}^{0}\right)^{\frac{1}{2}}+\frac{K_{p}^{0}}{\mu^{0}},
$$

(see (23) for the definition of $\left.\mu^{0}\right)$. Then, $K_{d}^{0}$ depends only on $\underline{\rho^{0}}, \overline{\rho^{0}},\left\|u^{0}\right\|_{H_{\sharp}^{1}}$ and, if $\tilde{T}_{0}<1$, there holds

$$
\int_{0}^{\tilde{T}_{0}}\left\|\partial_{x} u\right\|_{L_{\sharp}^{\infty}} \leq\left|\tilde{T}_{0}\right|^{\frac{1}{2}} K_{d}^{0}
$$

Proof. We recall first the classical embedding $H_{\sharp}^{1} \subset L_{\sharp}^{\infty}$ with the embedding inequality:

$$
\|v\|_{L_{\sharp}^{\infty}} \leq\left(\sqrt{L}+\frac{1}{\sqrt{L}}\right)\|v\|_{H_{\sharp}^{1}},
$$

Let now $T \leq \tilde{T}_{0}$. Due to (70), we have

$$
\int_{0}^{T} \int_{0}^{L}\left|\partial_{x} z\right|^{2} \leq K_{u}^{0} .
$$

Then, by construction, there holds:

$$
|z|^{2} \leq 2\left(|\mu|^{2}\left|\partial_{x} u\right|^{2}+|p|^{2}\right)
$$

Consequently, recalling (68), we obain:

$$
\begin{aligned}
\int_{0}^{T} \int_{0}^{L}|z|^{2} & \leq 2 \int_{0}^{T} \int_{0}^{L}|\mu|^{2}\left|\partial_{x} u\right|^{2}+2 \int_{0}^{T} \int_{0}^{L}|p|^{2} \\
& \leq 2 K_{\mu}^{0} \int_{0}^{T} \int_{0}^{L} \mu\left|\partial_{x} u\right|^{2}+2 T L\left|K_{p}^{0}\right|^{2} \\
& \leq 2 K_{\mu}^{0} \mathcal{E}_{0}^{c}+2 T L\left|K_{p}^{0}\right|^{2} .
\end{aligned}
$$

Finally, we have:

$$
\int_{0}^{T}\|z\|_{L_{\sharp}^{\infty}}^{2} \leq\left(\sqrt{L}+\frac{1}{\sqrt{L}}\right)^{2}\left(2 K_{\mu}^{0} \mathcal{E}_{0}^{c}+2 T L\left|K_{p}^{0}\right|^{2}+K_{u}^{0}\right) .
$$


and thus

$$
\int_{0}^{T}\|z\|_{L_{\sharp}^{\infty}} \leq \sqrt{T}\left(\sqrt{L}+\frac{1}{\sqrt{L}}\right)\left(2 K_{\mu}^{0} \mathcal{E}_{0}^{c}+2 T L\left|K_{p}^{0}\right|^{2}+K_{u}^{0}\right)^{\frac{1}{2}} .
$$

Then, we remark that

$$
\partial_{x} u=\frac{z+p}{\mu}, \quad \text { so that (with the bound (23)), } \quad\left|\partial_{x} u\right| \leq \frac{1}{\mu^{0}}|z|+\frac{p}{\mu^{0}}
$$

and :

$$
\int_{0}^{T}\left\|\partial_{x} u\right\|_{L_{\sharp}^{\infty}} \leq \frac{\sqrt{T}}{\mu^{0}}\left(\sqrt{L}+\frac{1}{\sqrt{L}}\right)\left(2 K_{\mu}^{0} \mathcal{E}_{0}^{c}+2 T L\left|K_{p}^{0}\right|^{2}+K_{u}^{0}\right)^{\frac{1}{2}}+\frac{T}{\mu^{0}} K_{p}^{0} .
$$

Hence, under the further restriction $T<1$, we obtain:

$$
\int_{0}^{T}\left\|\partial_{x} u\right\|_{L_{\sharp}^{\infty}} \leq \frac{\sqrt{T}}{\mu^{0}}\left[\left(\sqrt{L}+\frac{1}{\sqrt{L}}\right)\left(2 K_{\mu}^{0} \mathcal{E}_{0}^{c}+2 L\left|K_{p}^{0}\right|^{2}+K_{u}^{0}\right)^{\frac{1}{2}}+K_{p}^{0}\right]
$$

which yields the expected result setting $T=\tilde{T}_{0}$.

We now consider the continuity equation and derive bounds for $\rho$ :

Lemma 5. There exists $T_{0}^{\rho}$ depending only on $\underline{\rho^{0}}, \overline{\rho^{0}},\left\|u^{0}\right\|_{H_{\sharp}^{1}}$ for which, if we assume that $\tilde{T}_{0}<T_{0}^{\rho}$ then, there holds:

$$
\frac{2}{3} \underline{\rho}^{0}<\rho(t, x)<\frac{3}{2} \overline{\rho^{0}} \quad \forall(t, x) \in\left(0, \tilde{T}_{0}\right) \times \mathbb{R} .
$$

Proof. By standard arguments, we have that, for arbitrary $p \in[1, \infty[\cup]-\infty,-1[$ there holds:

$$
\frac{1}{p} \frac{\mathrm{d}}{\mathrm{d} t}\left[\int_{0}^{L}|\rho|^{p}\right]+\frac{1}{p} \int_{0}^{L} u \partial_{x}|\rho|^{p}=-\int_{0}^{L}|\rho|^{p} \partial_{x} u
$$

so that:

$$
\frac{\mathrm{d}}{\mathrm{d} t}\left[\int_{0}^{L}|\rho|^{p}\right] \leq|p-1| \int_{0}^{L}|\rho|^{p}\left|\partial_{x} u\right| \leq 2|p|\left\|\partial_{x} u\right\|_{L_{\sharp}^{\infty}} \int_{0}^{L}|\rho|^{p} .
$$

Consequently, there holds:

$$
\left(\int_{0}^{L}|\rho|^{p}\right)^{\frac{1}{p}} \leq\left(\int_{0}^{L}\left|\rho^{0}\right|^{p}\right)^{\frac{1}{p}} \exp \left(2 \int_{0}^{T}\left\|\partial_{x} u\right\|_{L_{\sharp}^{\infty}}\right) .
$$

In the limit $|p| \rightarrow \infty$ we thus have

$$
\begin{aligned}
& \|\rho(t, \cdot)\|_{L_{\sharp}^{\infty}} \leq \overline{\rho^{0}} \exp \left(2 \int_{0}^{T}\left\|\partial_{x} u\right\|_{L_{\sharp}^{\infty}}\right), \\
& \left\||\rho(t, \cdot)|^{-1}\right\|_{L_{\sharp}^{\infty}} \leq \frac{1}{\underline{\rho^{0}}} \exp \left(2 \int_{0}^{T}\left\|\partial_{x} u\right\|_{L_{\sharp}^{\infty}}\right) .
\end{aligned}
$$


Setting

$$
T_{0}^{\rho}:=\min \left(\frac{1}{2},\left|\frac{1}{2 K_{d}^{0}} \ln \left(\frac{3}{2}\right)\right|^{2}\right)
$$

(which has then the expected dependencies, see the definition of $K_{d}^{0}$ ), and assuming $\tilde{T}_{0}<$ $T_{0}^{\rho}<1$, we apply Lemma 4 on $\left(0, \tilde{T}_{0}\right)$ and obtain:

$$
\underline{\rho^{0}} \exp \left(-2\left|\tilde{T}_{0}\right|^{\frac{1}{2}} K_{d}^{0}\right) \leq \rho(t, x) \leq \overline{\rho^{0}} \exp \left(2\left|\tilde{T}_{0}\right|^{\frac{1}{2}} K_{d}^{0}\right),
$$

where

$$
\exp \left(2\left|\tilde{T}_{0}\right|^{\frac{1}{2}} K_{d}^{0}\right) \leq \exp \left(2 \sqrt{T_{0}^{\rho}} K_{d}\right) \leq \frac{3}{2}
$$

We conclude with deriving estimates for $u$ and $z$ :

Lemma 6. There exists $T_{0}^{u}>0$ depending only on $\underline{\rho^{0}}, \overline{\rho^{0}},\left\|u^{0}\right\|_{H_{\sharp}^{1}}$ for which, if we assume that $\tilde{T}_{0}<T_{0}^{u}$, there holds:

$$
\begin{aligned}
\sup _{t \in\left[0, \tilde{T}_{0}\right]}\left\|\partial_{x} u\right\|_{L_{\sharp}^{2}}^{2}+\int_{0}^{\tilde{T}_{0}}\left|\partial_{x} z\right|^{2} \mathrm{~d} & \\
\leq & \leq\left(\frac{8}{\mu^{0}}+36 \overline{\rho^{0}}\right)\left[\left\|\sqrt{\mu\left(\rho^{0}\right)} \partial_{x} u_{0}-\kappa\left(\rho^{0}\right)\right\|_{L_{\sharp}^{2}}^{2}+1+L\left|K_{\kappa}^{0}\right|^{2}\right] .
\end{aligned}
$$

Proof. The proof of this result is based on the use of a suitable multiplier for the momentum equation:

$$
\rho\left(\partial_{t} u+u \partial_{x} u\right)=\partial_{x}\left[\mu \partial_{x} u-p\right] \quad \text { a.e. on }(0, \infty) \times(0, L)
$$

Precisely, we introduce the following conventions:

- the operator $\mathbb{E}$ corresponds to the mean of an $L$-periodic $L^{1}$-function;

- the operator $\partial_{x}^{-1}$ corresponds to the periodic mean-free primitive of an $L$-periodic function of mean 0 . It maps $H_{\sharp}^{m}$ into $H_{\sharp}^{m+1}$ for arbitrary $m \in \mathbb{N}$ and admits a straightforward density extension for $m \in \mathbb{Z} \backslash \mathbb{N}$ (when $m \in \mathbb{Z} \backslash \mathbb{N}, H_{\sharp}^{m}$ stands for the dual of the subspace of $H_{\sharp}^{|m|}$ containing all functions with mean zero);

- throughout the proof, $C_{0}$ is a constant which depends only on $\underline{\rho^{0}}, \overline{\rho^{0}}$ and $\left\|u^{0}\right\|_{H_{\sharp}^{1}}$. It may vary between lines.

Then, we let $T \in\left(0, \tilde{T}_{0}\right)$ and we set:

$$
v=\partial_{t} u-\partial_{x}^{-1}\left[\partial_{t} \kappa-\mathbb{E}\left[\partial_{t} \kappa\right]\right] \quad \text { on }(0, T) .
$$

We recall that thanks to the continuity equation, there holds, for arbitrary $\beta \in C^{1}([0, \infty))$

$$
\partial_{t} \beta(\rho)+\partial_{x}(\beta(\rho) u)+\left(\beta^{\prime}(\rho) \rho-\beta(\rho)\right) \partial_{x} u=0 .
$$


Hence we have that $\kappa, \mu, p$ belong to the space $H^{1}\left(0, T ; L_{\sharp}^{2}\right) \cap C\left([0, T] ; C_{\sharp}\right) \cap L^{2}\left(0, T ; H_{\sharp}^{1}\right)$. Consequently, $v \in L^{2}\left((0, T) ; L_{\sharp}^{2}\right)$ and we have then:

$$
\int_{0}^{T} \int_{0}^{L} \rho\left(\partial_{t} u+u \partial_{x} u\right) v=\int_{0}^{T} \int_{0}^{L} \partial_{x}\left[\mu \partial_{x} u-p\right] v
$$

On the right-hand side, we note that we can approximate $u$ by projecting on Fourier series with a finite number of terms. This yields a sequence $u^{N}$ converging to $u$ in $H^{1}\left(0, T ; L_{\sharp}^{2}\right) \cap$ $C\left([0, T] ; H_{\sharp}^{1}\right) \cap L^{2}\left(0, T ; H_{\sharp}^{2}\right)$. Furthermore, the extension of $\partial_{x}^{-1}$ to negative sobolev spaces yields that:

$$
v=\partial_{x}^{-1}\left[\partial_{t} \partial_{x} u-\left(\partial_{t} \kappa-\mathbb{E}\left[\partial_{t} \kappa\right]\right)\right]
$$

Hence the following formal integration by parts that are valid for $L$-periodic trigonometric polynoms (with $\left.v^{N}=\partial_{t} u^{N}-\partial_{x}^{-1}\left[\partial_{t} \kappa-\mathbb{E}\left[\partial_{t} \kappa\right]\right]\right)$ :

$$
\begin{aligned}
\int_{0}^{L} \partial_{x}\left[\mu \partial_{x} u^{N}-p\right] v^{N}= & -\int_{0}^{L}\left[\mu \partial_{x} u^{N}-p\right] \partial_{x} v^{N} \\
= & -\int_{0}^{L} \mu\left[\partial_{x} u^{N}-\kappa\right] \partial_{t}\left[\partial_{x} u^{N}-\kappa\right]-\int_{0}^{L}\left[\mu \partial_{x} u^{N}-p\right] \mathbb{E}\left[\partial_{t} \kappa\right] \\
= & -\frac{1}{2} \frac{\mathrm{d}}{\mathrm{d} t} \int_{0}^{L} \mu\left|\partial_{x} u^{N}-\kappa\right|^{2}+\frac{1}{2} \int_{0}^{L} \partial_{t} \mu\left|\partial_{x} u^{N}-\kappa\right|^{2} \\
& -\mathbb{E}\left[\mu \partial_{x} u^{N}-p\right] \int_{0}^{L} \partial_{t} \kappa
\end{aligned}
$$

extend into:

$$
\begin{array}{r}
\int_{0}^{T} \int_{0}^{L} \partial_{x}\left[\mu \partial_{x} u-p\right] v=-\frac{1}{2}\left[\int_{0}^{L} \mu\left|\partial_{x} u-\kappa\right|^{2}\right]_{0}^{T}+\frac{1}{2} \int_{0}^{T} \int_{0}^{L} \partial_{t} \mu\left|\partial_{x} u-\kappa\right|^{2} \\
-\int_{0}^{T} \mathbb{E}\left[\mu \partial_{x} u-p\right] \int_{0}^{L} \partial_{t} \kappa
\end{array}
$$

This simplifies the RHS of (73), whereas, on the left-hand side, we have:

$$
\begin{aligned}
L H S & =\int_{0}^{T} \int_{0}^{L} \rho\left(\partial_{t} u+u \partial_{x} u\right)\left(\partial_{t} u-\partial_{x}^{-1}\left[\partial_{t} \kappa-\mathbb{E}\left[\partial_{t} \kappa\right]\right]\right) \\
& \geq \frac{1}{2} \int_{0}^{T} \int_{0}^{L} \rho\left|\partial_{t} u+u \partial_{x} u\right|^{2}-\int_{0}^{T} \int_{0}^{L} \rho\left|u \partial_{x} u\right|^{2}-\int_{0}^{T} \int_{0}^{L} \rho\left|\partial_{x}^{-1}\left[\partial_{t} \kappa-\mathbb{E}\left[\partial_{t} \kappa\right]\right]\right|^{2} .
\end{aligned}
$$


Finally, (73) reduces to ${ }^{2}$ :

$$
\begin{aligned}
\frac{1}{2}\left[\int_{0}^{L} \mu\left|\partial_{x} u-\kappa\right|^{2}\right]_{t=T}+\frac{1}{4 \overline{\rho^{0}}} \int_{0}^{T} \int_{0}^{L}\left|\partial_{x}\left[\mu \partial_{x} u-p\right]\right|^{2} \\
\leq \frac{1}{2}\left[\int_{0}^{L} \mu\left(\rho^{0}\right)\left|\partial_{x} u^{0}-\kappa\left(\rho^{0}\right)\right|^{2}\right]+\frac{1}{2} \int_{0}^{T} \int_{0}^{L} \partial_{t} \mu\left|\partial_{x} u-\kappa\right|^{2}-\int_{0}^{T} \mathbb{E}[z] \int_{0}^{L} \partial_{t} \kappa \\
+\int_{0}^{T} \int_{0}^{L} \rho\left|u \partial_{x} u\right|^{2}+\int_{0}^{T} \int_{0}^{L} \rho\left|\partial_{x}^{-1}\left[\partial_{t} \kappa-\mathbb{E}\left[\partial_{t} \kappa\right]\right]\right|^{2}, \\
\leq \frac{1}{2}\left[\int_{0}^{L} \mu\left(\rho^{0}\right)\left|\partial_{x} u^{0}-\kappa\left(\rho^{0}\right)\right|^{2}\right]+\frac{1}{2} I_{1}-I_{2}+I_{3}+I_{4} .
\end{aligned}
$$

We bound now $I_{1}, I_{2}, I_{3}, I_{4}$.

Applying (72) with $\beta=1 / \mu$, we have first:

$$
\begin{aligned}
I_{1} & =-\int_{0}^{T} \int_{0}^{L} \partial_{t}\left[\frac{1}{\mu}\right]\left|\mu \partial_{x} u-p\right|^{2} \\
& =\int_{0}^{T} \int_{0}^{L} \partial_{x}\left[\frac{u}{\mu}\right]\left|\mu \partial_{x} u-p\right|^{2}-\int_{0}^{T} \int_{0}^{L} \frac{\left(\mu^{\prime} \rho+\mu\right)}{\mu^{2}} \partial_{x} u\left|\mu \partial_{x} u-p\right|^{2} \\
& =-2 \int_{0}^{T} \int_{0}^{L} \frac{u}{\mu}\left[\mu \partial_{x} u-p\right] \partial_{x}\left[\mu \partial_{x} u-p\right]-\int_{0}^{T} \int_{0}^{L} \frac{\left(\mu^{\prime} \rho+\mu\right)}{\mu^{2}} \partial_{x} u\left|\mu \partial_{x} u-p\right|^{2} .
\end{aligned}
$$

Recalling that thanks to (68):

$$
\|u\|_{L_{\sharp}^{2}}^{2}=\int_{0}^{L}|u|^{2} \leq \frac{4}{\underline{\rho^{0}}} \int_{0}^{L} \frac{\rho|u|^{2}}{2} \leq \frac{4 \mathcal{E}_{c}^{0}}{\underline{\rho^{0}}},
$$

we obtain that, for arbitrary small $\varepsilon$ :

$$
\begin{aligned}
\left|I_{1}\right| & \leq C_{0} \int_{0}^{T}\left[\|u\|_{L_{\sharp}^{\infty}}\|z\|_{L_{\sharp}^{2}}\left\|\partial_{x} z\right\|_{L_{\sharp}^{2}}+\left\|\partial_{x} u\right\|_{L_{\sharp}^{\infty}}\|z\|_{L_{\sharp}^{2}}^{2}\right] \\
& \leq \frac{1}{8 \underline{\rho}^{0}} \int_{0}^{T}\left\|\partial_{x} z\right\|_{L_{\sharp}^{2}}^{2}+C_{0} \int_{0}^{T}\|z\|_{L_{\sharp}^{2}}^{2}\left[\|u\|_{L_{\sharp}^{2}}^{2}+\left\|\partial_{x} u\right\|_{L_{\sharp}^{2}}^{2}+\left\|\partial_{x} u\right\|_{L_{\sharp}^{\infty}}\right]
\end{aligned}
$$

Rewriting $z$ in terms of $\partial_{x} u$ and $p(\rho), \mu(\rho)$, we obtain finally that

$$
\left|I_{1}\right| \leq C_{0} \int_{0}^{T}\left(\left\|\partial_{x} u\right\|_{L_{\sharp}^{2}}^{2}+\left\|\partial_{x} u\right\|_{L_{\sharp}^{\infty}}+1\right) \int_{0}^{L} \mu\left|\partial_{x} u-\kappa\right|^{2}+\frac{1}{8 \underline{\rho^{0}}} \int_{0}^{T}\left\|\partial_{x} z\right\|_{L_{\sharp}^{2}}^{2} .
$$

Concerning $I_{2}=\int_{0}^{T} \int_{0}^{L} \mathbb{E}[z] \partial_{t} \kappa$, we have, applying (72):

$$
\partial_{t} \kappa+\partial_{x}(\kappa u)+\left(\kappa^{\prime} \rho-\kappa\right) \partial_{x} u=0,
$$

\footnotetext{
${ }^{2}$ Note that $\rho\left(\partial_{t} u+u \partial_{x} u\right)=\partial_{x}\left[\mu \partial_{x} u-p\right]$ and $\rho \leq 2 \overline{\rho^{0}}$
} 
so that

$$
\int_{0}^{L} \partial_{t} \kappa=-\int_{0}^{L}\left(\kappa^{\prime} \rho-\kappa\right) \partial_{x} u
$$

and consequently, with the same arguments as above:

$$
\left|I_{2}\right| \leq \int_{0}^{T} C_{0}\|z\|_{L_{\sharp}^{2}}\left\|\partial_{x} u\right\|_{L_{\sharp}^{2}} \leq C_{0} \int_{0}^{T}\left\|\partial_{x} u\right\|_{L_{\sharp}^{2}}^{2}+C_{0} \int_{0}^{T} \int_{0}^{L} \mu\left|\partial_{x} u-\kappa\right|^{2} .
$$

Concerning $I_{3}=\int_{0}^{T} \int_{0}^{L} \rho\left|u \partial_{x} u\right|^{2}$, we proceed as previously:

$$
\begin{aligned}
\left|I_{3}\right| & \leq C_{0} \int_{0}^{T}\|u\|_{L_{\sharp}^{\infty}}^{2} \int_{0}^{L}\left|\partial_{x} u\right|^{2} \\
& \leq C_{0} \int_{0}^{T}\left(1+\left\|\partial_{x} u\right\|_{L_{\sharp}^{2}}^{2}\right) \int_{0}^{L}\left|\partial_{x} u\right|^{2}
\end{aligned}
$$

Finally, expressing $\partial_{x} u$ in terms of $z$ and functions of $\rho$, there still exists a constant $C_{0}$ for which:

$$
\left|I_{3}\right| \leq C_{0} \int_{0}^{T}\left(1+\left\|\partial_{x} u\right\|_{L_{\sharp}^{2}}^{2}\right) \int_{0}^{L} \mu\left|\partial_{x} u-\kappa\right|^{2}+C_{0} \int_{0}^{T}\left(1+\left\|\partial_{x} u\right\|_{L_{\sharp}^{2}}^{2}\right) .
$$

Then, for $I_{4}$, we note as previously that:

$$
\partial_{t} \kappa-\mathbb{E}\left[\partial_{t} \kappa\right]=-\partial_{x}(\kappa u)-\left[\left(\kappa^{\prime} \rho-\kappa\right) \partial_{x} u-\mathbb{E}\left[\left(\kappa^{\prime} \rho-\kappa\right) \partial_{x} u\right]\right]
$$

Consequently, there holds:

$$
\partial_{x}^{-1}\left[\partial_{t} \kappa-\mathbb{E}\left[\partial_{t} \kappa\right]\right]=-[\kappa u-\mathbb{E}[\kappa u]]-w
$$

where

$$
w=\partial_{x}^{-1}\left[\left(\kappa^{\prime} \rho-\kappa\right) \partial_{x} u-\mathbb{E}\left[\left(\kappa^{\prime} \rho-\kappa\right) \partial_{x} u\right]\right] .
$$

A classical Poincaré-Wirtinger inequality yields that:

$$
\left\|\partial_{x}^{-1}\left[\partial_{t} \kappa-\mathbb{E}\left[\partial_{t} \kappa\right]\right]\right\|_{L_{\sharp}^{2}}^{2} \leq C_{0}\left[\|u\|_{L_{\sharp}^{2}}^{2}+\left\|\partial_{x} u\right\|_{L_{\sharp}^{2}}^{2}\right]
$$

Hence $I_{4}=\int_{0}^{T} \rho\left|\partial_{x}^{-1}\left[\partial_{t} \kappa-\mathbb{E}\left[\partial_{t} \kappa\right]\right]\right|^{2}$ satisfies:

$$
\left|I_{4}\right| \leq \int_{0}^{T} C_{0}\left(1+\left\|\partial_{x} u\right\|_{L_{\sharp}^{2}}^{2}\right) .
$$

Combining the computations (75)-(78) of $I_{1}, I_{2}, I_{3}, I_{4}$, we obtain finally that (74) reads:

$$
\begin{aligned}
& {\left[\int_{0}^{L} \mu\left|\partial_{x} u-\kappa\right|^{2}\right]_{t=T}+\frac{1}{8 \underline{\rho^{0}}} \int_{0}^{T} \int_{0}^{L}\left|\partial_{x} z\right|^{2}} \\
& \leq\left[\int_{0}^{L} \mu\left(\rho^{0}\right)\left|\partial_{x} u^{0}-\kappa\left(\rho^{0}\right)\right|^{2}\right]+\int_{0}^{T} f(t) \int_{0}^{L} \mu\left|\partial_{x} u-\kappa\right|^{2}+\int_{0}^{T} g(t)
\end{aligned}
$$

where

$$
f(t)=C_{0}\left(1+\left\|\partial_{x} u\right\|_{L_{\sharp}^{2}}^{2}+\left\|\partial_{x} u\right\|_{L^{\infty}(0, L)}\right) .
$$


and

$$
g(t)=C_{0}\left(1+\left\|\partial_{x} u\right\|_{L_{\sharp}^{2}}^{2}\right)
$$

On the one hand, we have that (we may assume $T<1$ without restriction so that (71) holds true):

$$
\begin{aligned}
\int_{0}^{T} f(t) \mathrm{d} t & \leq C_{0}\left(T+\int_{0}^{T}\left\|\partial_{x} u\right\|_{L^{\infty}(0, L)}+\left\|\partial_{x} u\right\|_{L_{\sharp}^{2}}^{2}\right) \\
& \leq C_{0}\left(T\left(1+K_{u}^{0}\right)+\sqrt{T} K_{d}^{0}\right) .
\end{aligned}
$$

Consequently, there exists $T_{0}^{u}<1$ depending only on $\underline{\rho^{0}}, \overline{\rho^{0}},\left\|u^{0}\right\|_{H_{\sharp}^{1}}$ such that:

$$
\exp \left(\int_{0}^{T_{0}^{u}} f(t) \mathrm{d} t\right) \leq 2
$$

Similarly we have:

$$
\int_{0}^{T} g(t) \mathrm{dt} \leq C_{0} T\left(1+K_{u}^{0}\right)
$$

Hence, restricting the size of $T_{0}^{u}$ if necessary, but keeping the same dependencies, we have that, for $T<T_{0}^{u}$ :

$$
\int_{0}^{T} g(t) \mathrm{dt} \leq \frac{1}{2}\left[\int_{0}^{L} \mu\left(\rho^{0}\right)\left|\partial_{x} u^{0}-\kappa\left(\rho^{0}\right)\right|^{2}+1\right] .
$$

Finally, by a standard application of the Gronwall lemma, we obtain then that, for arbitrary $T<T_{0}^{u}$, there holds:

$$
\begin{aligned}
\sup _{t \in[0, T]}\left\|\sqrt{\mu}\left(\partial_{x} u-\kappa\right)\right\|_{L_{\sharp}^{2}}^{2} & \leq \exp \left(\int_{0}^{T} f(t) \mathrm{dt}\right)\left(\left\|\sqrt{\mu\left(\rho^{0}\right)}\left(\partial_{x} u_{0}-\kappa\left(\rho^{0}\right)\right)\right\|_{L_{\sharp}^{2}}^{2}+2 \int_{0}^{T} g(s) \mathrm{d} s\right) \\
& \leq 4\left(\left\|\sqrt{\mu\left(\rho^{0}\right)} \partial_{x} u_{0}-\kappa\left(\rho^{0}\right)\right\|_{L_{\sharp}^{2}}^{2}+1\right)
\end{aligned}
$$

Consequently:

$$
\sup _{t \in[0, T]}\left\|\partial_{x} u\right\|_{L_{\sharp}^{2}}^{2} \leq \frac{8}{\mu^{0}}\left[\left\|\sqrt{\mu\left(\rho^{0}\right)} \partial_{x} u_{0}-\kappa\left(\rho^{0}\right)\right\|_{L_{\sharp}^{2}}^{2}+1+L\left|K_{p}^{0}\right|^{2}\right],
$$

and we also have:

$$
\begin{aligned}
\frac{1}{4 \overline{\rho^{0}}} \int_{0}^{T}\left|\partial_{x} z\right|^{2} \mathrm{~d} s \leq & \frac{1}{2}\left[\int_{0}^{L} \mu\left(\rho^{0}\right)\left|\partial_{x} u^{0}-\kappa\left(\rho^{0}\right)\right|^{2}\right] \\
& +4 \int_{0}^{T} f(t) \mathrm{dt}\left(\left\|\sqrt{\mu\left(\rho^{0}\right)}\left(\partial_{x} u_{0}-\kappa\left(\rho^{0}\right)\right)\right\|_{L_{\sharp}^{2}}^{2}+1\right)+\int_{0}^{T} g(s) \mathrm{d} s \\
\leq & 9\left(\left\|\sqrt{\mu\left(\rho^{0}\right)}\left(\partial_{x} u_{0}-\kappa\left(\rho^{0}\right)\right)\right\|_{L_{\sharp}^{2}}^{2}+1\right)
\end{aligned}
$$


Finally, we have indeed, that, for arbitrary $T \in\left[0, T_{0}^{u}\right)$ there holds:

$$
\begin{aligned}
& \sup _{t \in[0, T]}\left\|\partial_{x} u\right\|_{L_{\sharp}^{2}}^{2}+\int_{0}^{T}\left|\partial_{x} z\right|^{2} \mathrm{~d} s \\
& \leq\left(\frac{8}{\mu^{0}}+36 \overline{\rho^{0}}\right)\left[\left\|\sqrt{\mu\left(\rho^{0}\right)} \partial_{x} u_{0}-\kappa\left(\rho^{0}\right)\right\|_{L_{\sharp}^{2}}^{2}+1+L\left|K_{\kappa}^{0}\right|^{2}\right] .
\end{aligned}
$$

Combining Lemma 5 and Lemma 6, we obtain finally, that, for $T_{0}=\min \left(1, T_{0}^{\rho}, T_{0}^{u}\right) / 2$ we have $(\text { HDS })_{c}$ with

$$
K_{0}=36\left(\frac{1}{\mu^{0}}+\overline{\rho^{0}}\right)\left[\left\|\sqrt{\mu\left(\rho^{0}\right)} \partial_{x} u_{0}-\kappa\left(\rho^{0}\right)\right\|_{L_{\sharp}^{2}}^{2}+1+L\left|K_{\kappa}^{0}\right|^{2}\right] .
$$

4.3. Compactness argument : proof of main theorems. In this last section, we complete the proof of our mains results: Theorem 1 and Theorem 2 . We first remark that the proof of both results reduces to a study of compactness of HD solutions to (20)(21).

To complete the proof of Theorem 1, we remark that, given an initial data $\left(\rho^{0}, u^{0}\right) \in$ $L_{\sharp}^{\infty} \times H_{\sharp}^{1}$ we may approximate this initial data by a sequence $\left(\rho_{n}^{0}, u_{n}^{0}\right) \in L_{\sharp}^{\infty} \cap H_{\sharp}^{1}$ satisfying

$$
\underline{\rho^{0}} \leq \rho_{n}^{0} \leq \overline{\rho^{0}} \quad\left\|u_{n}^{0}\right\|_{H_{\sharp}^{1}} \leq\left\|u^{0}\right\|_{H_{\sharp}^{1}}, \quad \forall n \in \mathbb{N},
$$

and

$$
\rho_{n}^{0} \rightarrow \rho^{0} \text { in } L_{\sharp}^{1} \quad u_{n}^{0} \rightarrow u^{0} \text { in } H_{\sharp}^{1} .
$$

This can be done by a standard mollifying/projection argument. Then, the result in the previous section shows that there exists $T_{0}>0$ independant of $n \in \mathbb{N}$ for which there exists a HD solution $\left(\rho_{n}, u_{n}\right)$ to $(20)-(21)$ on $\left(0, T_{0}\right)$ associated with initial data $\left(\rho_{n}^{0}, u_{n}^{0}\right)$. Our objective is to prove that we can extract a subsequence of these HD solutions that converges to an HD solution to $(20)-(21)$ on $\left(0, T_{0}\right)$ associated with initial data $\left(\rho^{0}, u^{0}\right)$.

On the other hand, to complete the proof of Theorem 2, we consider a sequence of initial data $\left(\rho_{n}^{0}, u_{n}^{0}\right) \in L_{\sharp}^{\infty} \cap H_{\sharp}^{1}$. Under the assumptions of Theorem 2, there exists a constant $C_{0} \in(0, \infty)$ for which:

$$
\frac{1}{C_{0}} \leq \rho_{n}^{0} \leq C_{0} \quad\left\|u_{n}^{0}\right\|_{H_{\sharp}^{1}} \leq C_{0}, \quad \forall n \in \mathbb{N} .
$$

Assuming that Theorem 1 holds (that would result from a first application of the proof below), there exists $T_{0}>0$ independant of $n \in \mathbb{N}$ for which there exists a HD solution $\left(\rho_{n}, u_{n}\right)$ to $(20)-(21)$ on $\left(0, T_{0}\right)$ associated with initial data $\left(\rho_{n}^{0}, u_{n}^{0}\right)$. We aim then at studying if this sequence admits cluster point and to compute a system satisfied by these cluster points.

We first make precise the convergence of the initial data that we apply in Theorem 2 . We have the definition below: 
Definition 7. Given $\left(L, C_{0}\right)>0$ we call L-periodic Young-measure on $\mathbb{R} \times\left[0,2 C_{0}\right]$ any positive bounded measure $\mu$ on $R \times\left[0,2 C_{0}\right]$, L-periodic in the first variable, and satisfying:

$$
\langle\mu,(x, \xi) \mapsto \phi(x)\rangle=\int_{0}^{L} \phi(x) \mathrm{d} x .
$$

We denote $\mathcal{Y}_{\sharp}\left([0, L] \times\left[0,2 C_{0}\right]\right)$ the set of L-periodic Young measures.

This definition is an adaptation to the periodic framework of the definition of L. TARTAR [21]. It goes with several remarks:

(1) As $L, C_{0}$ will be fixed in what follows, we drop it in the notations for Young measures. From now on, we denote simply $\mathcal{Y}_{\sharp}$.

(2) As in the non-periodic case our Young measures form a closed subspace of the set of positive measures on $\mathbb{R} \times\left[0,2 C_{0}\right]$. As we work in an unbounded domain (in $x$ ), "weak $-*$ convergence" is understood locally (see [21, Section 2] for more details).

(3) there holds $\mathcal{Y}_{\sharp} \subset\left[L^{1}\left((0,2 L) ; C\left(\left[0,2 C_{0}\right]\right)\right)\right]^{*}$. Indeed, if $\phi \in C_{c}\left((0,2 L) \times\left[0,2 C_{0}\right]\right)$, we have then that $|\phi(x, \xi)| \leq\|\phi(x, \cdot)\|_{C\left(\left[0,2 C_{0}\right]\right)}$ and, by the positivity of $\nu$ and (83):

$$
|\langle\nu, \phi\rangle| \leq \int_{0}^{2 L}\|\phi(x, \cdot)\|_{C\left(\left[0,2 C_{0}\right]\right)} \mathrm{d} x .
$$

The embedding property yields then from the fact that $C_{c}\left((0,2 L) \times\left[0,2 C_{0}\right]\right)$ is dense in $L^{1}\left((0,2 L) ; C\left(\left[0,2 C_{0}\right]\right)\right)$,

(4) given $\rho \in L_{\sharp}^{\infty}$ such that $\|\rho\|_{L_{\sharp}^{\infty}} \leq 2 C_{0}$, we define $\nu_{\rho} \in \mathcal{Y}_{\sharp}$ by

$$
\left\langle\nu_{\rho}, \beta\right\rangle=\int_{0}^{L} \beta(x, \rho(x)) \mathrm{d} x, \quad \forall \beta \in C_{c}\left(\mathbb{R} \times\left[0,2 C_{0}\right]\right) .
$$

In the frame of Theorem 2 we assume that there exists $k \in \mathbb{N}$ and $\left(\alpha_{i}^{0}, \rho_{i}^{0}\right) \in\left[L_{\sharp}^{\infty}\right]^{2 k}$ for which there holds:

- $\alpha_{i}^{0} \geq 0$ a.e. for $1 \leq i \leq k$ with $\sum_{i=1}^{k} \alpha_{i}^{0}=1$, a.e.,

- $1 / C_{0} \leq \rho_{i}^{0} \leq C^{0}$ a.e. for $1 \leq i \leq k$,

- $\nu_{\rho_{n}^{0}} \rightarrow \nu^{0}=\sum_{i=1}^{k} \alpha_{i} \delta_{\xi=\rho_{i}}$.

Given the topology on measures, the last item is equivalent to:

$$
\beta\left(\rho_{n}\right) \rightarrow \sum_{i=1}^{k} \alpha_{i} \beta\left(\rho^{i}\right) \text { in } L_{\sharp}^{\infty}-w *, \quad \forall \beta \in C\left(\left[0,2 C_{0}\right]\right) .
$$

We recall in particular, that, if $\left\|\rho_{n}^{0}\right\|_{L_{\sharp}^{\infty}} \leq C_{0}$ for arbitrary $n \in \mathbb{N}$ and $\rho_{n}^{0} \rightarrow \rho^{0}$ in $L_{\sharp}^{1}$, there holds:

$$
\nu_{\rho_{n}} \rightarrow \nu_{\rho} \quad \text { in } \quad \mathcal{Y}_{\sharp}-w *
$$


Hence, the compactness study leading to Theorem $\mathbf{1}$ is a particular case of the proof of Theorem 2 (in the case $k=1$ ). We complete thus the study by the proof of Theorem 2 only.

So, we have now a uniform time $T_{0}>0$, and a sequence $\left(\rho_{n}, u_{n}\right)$ of HD solutions associated with data $\left(\rho_{n}^{0}, u_{n}^{0}\right)$ satisfying $(82)$. Thanks to these uniform bounds (HDS) $)_{c}$ yields:

- $\rho_{n}$ is bounded in $L^{\infty}\left(0, T_{0} ; L_{\sharp}^{\infty}\right)$ (from above and by below), and so do $\mu_{n}:=\mu\left(\rho_{n}\right), p_{n}:=$ $p\left(\rho_{n}\right)$ and $\kappa_{n}:=\kappa\left(\rho_{n}\right)$,

- $u_{n}$ is bounded in $L^{\infty}\left(0, T_{0} ; H_{\sharp}^{1}\right)$,

- $z_{n}:=\mu_{n} \partial_{x} u_{n}-p_{n}$ is bounded in $L^{2}\left(0, T_{0} ; H_{\sharp}^{1}\right)$.

Lemma 4 together with standard computations imply then that

- $\partial_{x} u_{n}$ is bounded in $L^{1}\left(0, T_{0} ; L_{\sharp}^{\infty}\right) \cap L^{\infty}\left(0, T_{0} ; L_{\sharp}^{2}\right)$.

We have thus, up to the extraction of a subsequence (that we do not relabel for conciseness):

- $\rho_{n} \rightarrow \rho, p_{n} \rightarrow p^{\infty}, \mu_{n} \rightarrow \mu^{\infty}$ and $\kappa_{n} \rightarrow \kappa^{\infty}$ in $L^{\infty}\left(0, T_{0} ; L_{\sharp}^{\infty}\right)-w *$,

- $u_{n} \rightarrow u$ in $L^{\infty}\left(0, T_{0} ; H_{\sharp}^{1}\right)-w *$ with $\partial_{x} u \in L^{1}\left(0, T_{0} ; L_{\sharp}^{\infty}\right)$

- $z_{n} \rightarrow z^{\infty}$ in $L^{2}\left(0, T_{0} ; H_{\sharp}^{1}\right)-w$.

Furthermore, introducing:

$$
\underline{\rho}_{\infty}:=\liminf \left(\inf _{\left(0, T_{0}\right) \times \mathbb{R}} \rho_{n}\right)_{n \in \mathbb{N}}, \quad \bar{\rho}_{\infty}:=\limsup \left(\sup _{\left(0, T_{0}\right) \times \mathbb{R}} \rho_{n}\right)_{n \in \mathbb{N}},
$$

classical weak convergence arguments also yield that,

- for a.e. $(t, x) \in\left(0, T_{0}\right) \times(\mathbb{R} / L \mathbb{Z})$ there holds:

$$
\underline{\rho}_{\infty} \leq \rho \leq \bar{\rho}_{\infty}
$$

- there exists a constant $K_{0}$ depending only on $C_{0}$ and $\sup _{n \in \mathbb{N}}\left\|u_{n}^{0}\right\|_{H_{\sharp}^{1}}$ for which

$$
\sup _{t \in\left(0, T_{0}\right)}\|u(t, \cdot)\|_{H_{\sharp}^{1}}+\int_{0}^{T_{0}}\left\|z^{\infty}(t, \cdot)\right\|_{H_{\sharp}^{1}} \leq K_{0} .
$$

4.3.1. Convergence of momentum equation. We want now to pass to the limit in the momentum equation satisfied by $\rho_{n}$ and $u_{n}$. To this end, we first obtain strong-compactness for two quantities. We have:

Lemma 8. Up to the extraction of a subsequence, we have that

$$
u_{n} \rightarrow u \text { in } C\left(\left[0, T_{0}\right] ; L_{\sharp}^{2}\right) .
$$

Proof. We already have that $u_{n}$ is bounded in $C\left(\left[0, T_{0}\right] ; L_{\sharp}^{2}\right) \cap L^{\infty}\left(0, T_{0} ; H_{\sharp}^{1}\right)$ where $H_{\sharp}^{1} \subset L_{\sharp}^{2}$ is compact. Furthermore, we have from the continuity equation that:

$$
\partial_{t} u_{n}=-u_{n} \partial_{x} u_{n}+\frac{1}{\rho_{n}} \partial_{x} z_{n} .
$$


Consequently:

$$
\left\|\partial_{t} u_{n}\right\|_{L^{2}\left(0, T_{0} ; L_{\sharp}^{2}\right)} \leq\left\|u_{n}\right\|_{L^{\infty}\left(0, T_{0} ; L_{\sharp}^{\infty}\right)}\left\|u_{n}\right\|_{L^{2}\left(0, T_{0} ; H_{\sharp}^{1}\right)}+\left\|\left|\rho_{n}\right|^{-1}\right\|_{L^{\infty}\left(0, T_{0} ; L_{\sharp}^{\infty}\right)}\left\|\partial_{x} z_{n}\right\|_{L^{2}\left(0, T_{0} ; L_{\sharp}^{2}\right)} .
$$

But, the bounds claimed above and the embedding $H_{\sharp}^{1} \subset L_{\sharp}^{\infty}$ yield that the right-hand side of this inequality is bounded uniformly in $n \in \mathbb{N}$. Consequently, we have that $u_{n}$ is uniformly equicontinuous in $C\left(\left[0, T_{0}\right] ; L_{\sharp}^{2}\right)$ and we may extract a strongly converging subsequence.

Remark. We can then prove that $\rho^{n}\left|u^{n}\right|^{2} \rightarrow \rho|u|^{2}$ (in $L^{2}((0, T) \times(\mathbb{R} / L \mathbb{Z}))-w$ for instance) and, if $\rho_{n}^{0}$ converges strongly to $\rho^{0}$, classical arguments on the dissipation estimate satisfied by $\left(\rho^{n}, u^{n}\right)$ imply that for a.e. $t \in\left(0, T_{0}\right)$ there holds:

$$
\int_{0}^{L}\left[\frac{\rho(t, \cdot)|u(t, \cdot)|^{2}}{2}+q^{\infty}\right]+\int_{0}^{t} \int_{0}^{L} \mu\left|\partial_{x} u\right|^{2} \leq \int_{0}^{L}\left[\frac{\rho^{0}\left|u^{0}\right|^{2}}{2}+q^{0}\right]
$$

where $q^{0}=q\left(\rho^{0}\right)$.

Second, we state the equivalent result to the viscous-flux lemma that was crucial to the proof by P.-L. Lions [15] and by E. Feireisl, A. Novotný and H. Petzeltová [9] to obtain existence of global weak solutions to compressible Navier-Stokes systems:

Lemma 9. Let $\beta \in C^{1}((0, \infty))$ then, up to the extraction of a subsequence, we have that

$$
\begin{aligned}
\beta\left(\rho_{n}\right) \rightarrow \beta^{\infty} & \text { in } L^{\infty}\left(0, T_{0} ; L_{\sharp}^{\infty}\right)-w *, \\
\beta\left(\rho_{n}\right) z_{n} \rightarrow \beta^{\infty} z^{\infty} & \text { in } L^{2}\left(\left(0, T_{0}\right) \times \mathbb{R} / L \mathbb{Z}\right)-w .
\end{aligned}
$$

Proof. Under the assumptions of this lemma (and keeping the conventions of the previous section for the operator $\partial_{x}^{-1}$ ), we set:

$$
\beta_{n}=\beta\left(\rho_{n}\right), \quad w_{n}=\partial_{x}^{-1}\left[\beta_{n}-\mathbb{E}\left[\beta_{n}\right]\right] .
$$

Then, $\beta_{n}$ and $w_{n}$ are bounded respectively in $C\left(\left[0, T_{0}\right] ; L_{\sharp}^{2}\right) \cap L^{\infty}\left(\left(0, T_{0}\right) \times \mathbb{R} / L \mathbb{Z}\right)$ and $C\left(\left[0, T_{0}\right] ; H_{\sharp}^{1}\right)$. In particular, we may extract a subsequence s.t. $\beta\left(\rho^{n}\right)$ and $\beta\left(\rho_{n}\right) z_{n}$ converge respectively in $L^{\infty}\left(\left(0, T_{0}\right) \times \mathbb{R} / L \mathbb{Z}\right)-w *$ and $L^{2}\left(\left(0, T_{0}\right) \times \mathbb{R} / L \mathbb{Z}\right)-w$. We denote $\beta^{\infty}$ the weak $-*$ limit of $\beta\left(\rho_{n}\right)$. Furthermore, there holds:

$$
\partial_{t} w_{n}=\partial_{x}^{-1}\left[\partial_{t} \beta_{n}-\mathbb{E}\left[\partial_{t} \beta_{n}\right]\right]
$$

where, as previously:

$$
\begin{aligned}
\partial_{t} \beta_{n} & =-\partial_{x}\left(\beta_{n} u_{n}\right)-\left(\beta^{\prime}\left(\rho_{n}\right) \rho_{n}-\beta_{n}\right) \partial_{x} u_{n} \in L^{\infty}\left(0, T_{0} ; H_{\sharp}^{-1}\right) \\
\partial_{t} \mathbb{E}\left[\beta_{n}\right] & =-\mathbb{E}\left[\left(\beta^{\prime}\left(\rho_{n}\right) \rho_{n}-\beta_{n}\right) \partial_{x} u_{n}\right] \in L^{\infty}\left(0, T_{0}\right) .
\end{aligned}
$$

Consequently :

$$
\partial_{t} w_{n}=-\left(\beta_{n} u_{n}-\mathbb{E}\left[\beta_{n} u_{n}\right]\right)-\partial_{x}^{-1}\left[\left(\beta^{\prime}\left(\rho_{n}\right) \rho_{n}-\beta_{n}\right) \partial_{x} u_{n}-\mathbb{E}\left[\left(\beta^{\prime}\left(\rho_{n}\right) \rho_{n}-\beta_{n}\right) \partial_{x} u_{n}\right]\right]
$$


and repeating the computations for $I_{4}$ in the previous paragraph, we obtain that $\partial_{t} w_{n}$ is also bounded in $L^{\infty}\left(0, T_{0} ; L_{\sharp}^{2}\right)$. We may again extract a subsequence we do not relabel such that:

$$
w_{n} \rightarrow w^{\infty}=\partial_{x}^{-1}\left[\beta^{\infty}-\mathbb{E}\left[\beta^{\infty}\right]\right] \text { in } C\left(\left[0, T_{0}\right] ; L_{\sharp}^{2}\right)
$$

(as this is the only possible limit). Consequently also $\mathbb{E}\left[\beta_{n}\right]$ is bounded in $W^{1, \infty}\left(\left(0, T_{0}\right)\right)$ so that we may extract a subsequence for which $\mathbb{E}\left[\beta_{n}\right] \rightarrow \mathbb{E}\left[\beta^{\infty}\right]$ in $C\left(\left[0, T_{0}\right]\right)$.

For any $n \in \mathbb{N}$ and $\varphi \in C_{c}^{\infty}\left(\left(0, T_{0}\right) \times(0, L)\right)$ we have then:

$$
\int_{0}^{T_{0}} \int_{0}^{L} \beta_{n} z_{n} \varphi=\int_{0}^{T_{0}} \int_{0}^{L} \partial_{x} w_{n} z_{n} \varphi+\int_{0}^{T_{0}} \int_{0}^{L} \mathbb{E}\left[\beta_{n}\right] z_{n} \varphi
$$

On the one-hand, we have:

$$
\begin{aligned}
\int_{0}^{T_{0}} \int_{0}^{L} \mathbb{E}\left[\beta_{n}\right] z_{n} \varphi & =\frac{1}{L} \int_{0}^{T_{0}} \int_{0}^{L} \beta_{n} \int_{0}^{L} z_{n} \varphi \\
& \longrightarrow \frac{1}{L} \int_{0}^{T_{0}} \int_{0}^{L} \beta^{\infty} \int_{0}^{L} z^{\infty} \varphi
\end{aligned}
$$

due to the strong convergence of $\left(\mathbb{E}\left(\beta_{n}\right)\right)_{n \in \mathbb{N}}$ in $C\left(\left[0, T_{0}\right]\right)$ and the weak convergence of $\left(\int_{0}^{L} z_{n} \varphi\right)_{n \in \mathbb{N}}$ in $L^{2}\left(\left(0, T_{0}\right)\right)$.

On the other hand, there holds:

$$
\int_{0}^{T_{0}} \int_{0}^{L} \partial_{x} w_{n} z_{n} \varphi=-\int_{0}^{T_{0}} \int_{0}^{L} w_{n} \varphi \partial_{x} z_{n}-\int_{0}^{T_{0}} \int_{0}^{L} w_{n} z_{n} \partial_{x} \varphi
$$

Combining, the strong convergence of $w_{n}$ in $C\left(\left[0, T_{0}\right] ; L_{\sharp}^{2}\right)$ and the weak convergence of $z_{n}$ in $L^{2}\left(0, T_{0} ; H_{\sharp}^{1}\right)$ we also get:

$$
\int_{0}^{T_{0}} \int_{0}^{L} \partial_{x} w_{n} z_{n} \varphi \longrightarrow-\int_{0}^{T_{0}} \int_{0}^{L} w^{\infty} \varphi \partial_{x} z^{\infty}-\int_{0}^{T_{0}} \int_{0}^{L} w^{\infty} z^{\infty} \partial_{x} \varphi
$$

These computations entail finally that

$$
\begin{aligned}
\lim _{n \rightarrow \infty} \int_{0}^{T_{0}} \int_{0}^{L} \beta_{n} z_{n} \varphi & =-\int_{0}^{T_{0}} \int_{0}^{L} w^{\infty} \varphi \partial_{x} z^{\infty}-\int_{0}^{T_{0}} \int_{0}^{L} w^{\infty} z^{\infty} \partial_{x} \varphi+\int_{0}^{T_{0}} \int_{0}^{L} \mathbb{E}\left[\beta^{\infty}\right] w^{\infty} \varphi \\
& =\int_{0}^{T_{0}} \int_{0}^{L} \beta^{\infty} z^{\infty} \varphi .
\end{aligned}
$$

This completes the proof.

We can now pass to the limit in the equations satisfied by $\left(\rho_{n}, u_{n}\right)$.

Proposition 10. We have in $\mathcal{D}^{\prime}\left(\left(0, T_{0}\right) \times \mathbb{R}\right)$ :

$$
\partial_{t}(\rho u)+\partial_{x}\left(\rho u^{2}\right)=\partial_{x}\left[m^{\infty}\left(\partial_{x} u-\kappa^{\infty}\right)\right] .
$$


where

$$
m^{\infty}=\left[\lim \frac{1}{\mu\left(\rho_{n}\right)}\right]^{-1}
$$

Proof. We recall that, for any given $n \in \mathbb{N}$ there holds:

$$
\partial_{t}\left(\rho_{n} u_{n}\right)+\partial_{x}\left(\rho_{n}\left|u_{n}\right|^{2}\right)=\partial_{x} z_{n} .
$$

Combining the weak convergences of $\rho_{n}$ and $u_{n}$ and the strong convergence of $u_{n}$, we obtain that

- $\rho_{n} u_{n} \rightarrow \rho u$ in $L^{2}\left(\left(0, T_{0}\right) \times(\mathbb{R} / L \mathbb{Z})\right)-w$,

- $u_{n} \rightarrow u$ in $L^{4}\left(\left(0, T_{0}\right) \times(\mathbb{R} / L \mathbb{Z})\right)$ or $\left|u_{n}\right|^{2} \rightarrow u^{2}$ in $L^{2}\left(\left(0, T_{0}\right) \times(\mathbb{R} / L \mathbb{Z})\right)$,

- $\rho_{n}\left|u_{n}\right|^{2} \rightarrow \rho u^{2}$ in $L^{2}\left(\left(0, T_{0}\right) \times(\mathbb{R} / L \mathbb{Z})\right)-w$.

This enables to pass to the limit in the right-hand side:

$$
\partial_{t}\left(\rho_{n} u_{n}\right)+\partial_{x}\left(\rho_{n}\left|u_{n}\right|^{2}\right) \rightarrow \partial_{t}(\rho u)+\partial_{x}\left(\rho u^{2}\right) \text { in } \mathcal{D}^{\prime}\left(\left(0, T_{0}\right) \times \mathbb{R}\right)
$$

On the right-hand side we have that $z_{n} \rightarrow z^{\infty}$ so that:

$$
\partial_{t}(\rho u)+\partial_{x}\left(\rho u^{2}\right)=\partial_{x} z^{\infty} .
$$

It remains to compute $z$ in terms of $\rho$ and $u$. We have, for fixed $n \in \mathbb{N}$ :

$$
\partial_{x} u_{n}=\frac{z_{n}}{\mu_{n}}+\kappa\left(\rho_{n}\right)
$$

Passing to the limit in this identity (in $L^{2}\left(\left(0, T_{0}\right) \times(\mathbb{R} / L \mathbb{Z})\right)-w$ for instance), we get, thanks to the previous lemma:

$$
\partial_{x} u=\lim \left[\frac{1}{\mu\left(\rho_{n}\right)}\right] z^{\infty}+\kappa^{\infty} \text { or } z^{\infty}=m^{\infty}\left(\partial_{x} u-\kappa^{\infty}\right) .
$$

This ends the proof of this proposition.

As classical in these compactness arguments, the main difficulty now is to find a relation between $\mu^{\infty}, \kappa^{\infty}$ and $\rho$. In full generality, this is not possible: the operators "lim" and the operator "composition by a continuous function $\beta$ " do not commute. To analyze more precisely the commutators, we apply Young-measure theory.

4.3.2. Compactness of Young measures. For a given $n \in \mathbb{N}$ we introduce the young measure $\nu_{n}:=\nu_{\rho_{n}}$. From the regularity $\rho_{n} \in C\left(\left[0, T_{0}\right] ; L_{\sharp}^{1}\right)$ we deduce that $\nu_{n} \in C\left(\left[0, T_{0}\right] ; \mathcal{Y}_{\sharp}-w *\right)$. We state then

Proposition 11. There exists a subsequence we do not relabel such that $\nu_{n} \rightarrow \nu$ in $C\left(\left[0, T_{0}\right] ; \mathcal{Y}_{\sharp}-w *\right)$ Furthermore, $\nu$ is a solution to:

$$
\partial_{t} \nu+\partial_{x}(\nu u)-\left(\partial_{\xi}\left(\xi \frac{\nu}{\mu(\xi)}\right)+\frac{\nu}{\mu(\xi)}\right) z^{\infty}-\left(\partial_{\xi}\left(\frac{\xi p(\xi) \nu}{\mu(\xi)}\right)+\frac{\nu p(\xi)}{\mu(\xi)}\right)=0
$$


in $\mathcal{D}^{\prime}\left(\left(0, T_{0}\right) \times \mathbb{R} \times\left(0,2 C_{0}\right)\right)$ with initial condition:

$$
\nu(0, \cdot)=\sum_{i=1}^{k} \alpha_{i}^{0} \delta_{\rho_{i}^{0}}
$$

Proof. By construction, $\nu_{n} \in C\left(\left[0, T_{0}\right] ; \mathcal{Y}_{\sharp}-w *\right)$ and is a bounded sequence in this space. Then, we rewrite $(72)$ :

$$
\partial_{t} \nu_{n}+\partial_{x}\left(\nu_{n} u_{n}\right)-\left(\partial_{\xi}\left(\xi \nu_{n}\right)+\nu_{n}\right) \partial_{x} u_{n}=0 \quad \text { in } \mathcal{D}^{\prime}\left(\left(0, T_{0}\right) \times \mathbb{R} \times\left(0,2 C_{0}\right)\right) .
$$

For arbitrary $\phi \in C_{c}^{\infty}\left(\mathbb{R} \times\left(0,2 C_{0}\right)\right)$ there holds:

$$
\partial_{t}\left\langle\nu_{n}, \phi\right\rangle=\left\langle\nu_{n}, u_{n} \partial_{x} \phi\right\rangle+\left\langle\nu_{n}, \phi \partial_{x} u_{n}\right\rangle-\left\langle\nu_{n}, \xi \partial_{x} u_{n} \partial_{\xi} \phi\right\rangle .
$$

We recall here that $u_{n}$ is bounded in $L^{\infty}\left((0, T) ; C_{\sharp}\right)$ and that $\partial_{x} u_{n}$ is bounded also in the space $L^{1}\left(0, T ; L_{\sharp}^{\infty}\right)$. Hence, for arbitrary $\phi \in C_{c}^{\infty}\left(\mathbb{R} \times\left(0,2 C_{0}\right)\right)$ we have that $\left(\left\langle\nu_{n}, \phi\right\rangle\right)_{n \in \mathbb{N}}$ is relatively compact in $C\left(\left[0, T_{0}\right]\right)$.

As the weak-* convergence on $\mathcal{Y}_{\sharp}$ measures only the weak-* convergences in all the $C_{c}\left((-N, N) \times\left(0,2 C_{0}\right)\right)^{*}\left(\right.$ for $N \in \mathbb{N}$, since $\nu_{n}$ has support in $\left.\left.\mathbb{R} \times\left[1 / C_{0}, C_{0}\right]\right)\right)$ which admits a denumerable dense set of functions belonging to $C_{c}^{\infty}\left((-N, N) \times\left(0,2 C_{0}\right)\right)$, we may apply a classical argument to obtain that, up to the extraction of a subsequence, $\nu_{n}$ converges in $C\left(\left[0, T_{0}\right] ; \mathcal{Y}_{\sharp}-w *\right)$.

Then, we rewrite equivalently (89) as:

$$
\partial_{t} \nu_{n}+\partial_{x}\left(\nu_{n} u_{n}\right)-\left(\partial_{\xi}\left(\xi \frac{\nu_{n}}{\mu(\xi)}\right)+\frac{\nu_{n}}{\mu(\xi)}\right) z_{n}-\left(\partial_{\xi}\left(\frac{\xi p(\xi) \nu_{n}}{\mu(\xi)}\right)+\frac{\nu_{n} p(\xi)}{\mu(\xi)}\right)=0
$$

Combining the weak convergence of $\nu_{n}, z_{n}$, the strong convergence of $u_{n}$ and Lemma 9 we may pass to the limit in this equation and obtain (87).

To end the proof of Theorem 1 we remark that (87)-(88) enters the framework of Appendix A. Indeed, we rewrite (87) as

$$
\partial_{t} \nu+\partial_{x}\left(\nu u_{x}\right)+\partial_{\xi}\left(\nu u_{\xi}\right)+g \nu=0
$$

with, thanks to the previous arguments $u_{x}=u(t, x) \in C\left(\left[0, T_{0}\right] \times \mathbb{R} / L \mathbb{Z}\right)$ s.t.:

$$
\partial_{x} u_{x}=\frac{z^{\infty}}{m^{\infty}}+\kappa^{\infty} \in L^{1}\left(0, T_{0} ; L_{\sharp}^{\infty}\right), \quad \partial_{\xi} u_{x}=0 .
$$

and (note that $\left.z^{\infty} \in L^{2}\left(0, T_{0} ; H^{1}(\mathbb{R} / L \mathbb{Z})\right) \subset L^{1}\left(0, T_{0} ; C(\mathbb{R} / L \mathbb{Z})\right)\right)$ :

$$
u_{\xi}=-\left(\frac{\xi}{\mu(\xi)} z^{\infty}(t, x)+\frac{\xi p(\xi)}{\mu(\xi)}\right) \in L^{1}\left(0, T_{0} ; C\left(\mathbb{R} / L \mathbb{Z} \times\left[0,2 C_{0}\right]\right)\right)
$$

such that:

$$
\begin{gathered}
\partial_{\xi} u_{\xi} \in L^{1}\left(0, T_{0} ; L^{\infty}\left(\mathbb{R} / L \mathbb{Z} \times\left(0,2 C_{0}\right)\right)\right) ; \\
\int_{0}^{T} \int_{0}^{L} \sup _{\xi \in\left[0,2 C_{0}\right]}\left|\partial_{x} u_{\xi}(t, x, \xi)\right| \mathrm{d} \xi \mathrm{d} x<\infty .
\end{gathered}
$$


Finally, we have:

$$
g=-\left(\frac{z^{\infty}(t, x)}{\mu(\xi)}+\frac{p(\xi)}{\mu(\xi)}\right) \in L^{1}\left(0, T_{0} ; C\left(\mathbb{R} / L \mathbb{Z} \times\left[0,2 C_{0}\right]\right)\right)
$$

such that:

$$
\begin{gathered}
\partial_{\xi} g \in L^{1}\left(0, T_{0} ; L^{\infty}\left(\mathbb{R} / L \mathbb{Z} \times\left(0,2 C_{0}\right)\right)\right), \\
\int_{0}^{T} \int_{0}^{L} \sup _{\xi \in\left[0,2 C_{0}\right]}\left|\partial_{x} g(t, x, \xi)\right| \mathrm{d} \xi \mathrm{d} x<\infty .
\end{gathered}
$$

Hence, Appendix A ensures that $\nu$ is the unique solution to (87)-(88) and that it writes as a convex combination of $k$ Dirac measures. Plugging formally $\nu=\sum_{i=1}^{k} \alpha_{i} \delta_{\xi=\rho_{i}}$ in (87)(88) we get that the $\left(\alpha_{i}, \rho_{i}\right)$ are solutions of the expected pde system. We note that these equations are actually satisfied by construction (see the proof of Lemma 13). This ends the proof of Theorem 2.

Let mention that, in the particular case $k=1$, we recover that $\alpha_{1}=1$ and that $\left(\rho^{1}, u\right)=$ $(\rho, u)$ satisfies (20)-(21). We also have that (84)-(86)-(85) imply that (30)-(31)-(32) holds true on $\left(0, T_{0}\right)$. This completes the proof of Theorem 1.

\section{Appendix A. Complementary Result on transport equation}

In this section we consider periodic young-measures solution to the transport equation:

$$
\partial_{t} \nu+\operatorname{div}(u \nu)+g \nu=0,
$$

in $\mathcal{D}^{\prime}((0, T) \times \mathbb{R} \times(0, M))$, with initial condition:

$$
\nu(0, \cdot)=\nu^{0} .
$$

For legibility, we turn to notations $\left(x_{1}, x_{2}\right)$ for space variables and $u=\left(u_{1}, u_{2}\right)$ for velocityfields. Throughout this appendix, we assume that this velocity-field satisfies:

- $u_{1} \in L^{1}(0, T ; C(\mathbb{R} / L \mathbb{Z} \times[0, M]))$ with:

$$
\begin{aligned}
& \partial_{1} u_{1} \in L^{1}\left((0, T) ; L^{\infty}((\mathbb{R} / L \mathbb{Z}) \times(0, M))\right) ; \\
& \partial_{2} u_{1}=0 \quad \text { a.e. }
\end{aligned}
$$

- $u_{2} \in L^{1}((0, T) ; C((\mathbb{R} / L \mathbb{Z}) \times[0, M]))$ with:

$$
\begin{gathered}
\int_{0}^{T} \int_{0}^{L} \sup _{x_{2} \in[0, M]}\left|\partial_{1} u_{2}\left(t, x_{1}, x_{2}\right)\right| \mathrm{d} x_{1} \mathrm{~d} t<\infty \\
\partial_{2} u_{2} \in L^{1}\left((0, T) ; L^{\infty}((\mathbb{R} / L \mathbb{Z}) \times(0, M))\right) .
\end{gathered}
$$

As for the source term $g$, we assume that

- $g \in L^{1}\left(0, T ; C^{0}(\mathbb{R} / L \mathbb{Z} \times[0, M])\right)$ with 


$$
\begin{gathered}
\int_{0}^{T} \int_{0}^{L} \sup _{x_{2} \in[0, M]}\left|\partial_{1} g\left(t, x_{1}, x_{2}\right)\right| \mathrm{d} x_{1} \mathrm{~d} t<\infty \\
\partial_{2} g \in L^{1}\left((0, T) ; L^{\infty}((\mathbb{R} / L \mathbb{Z}) \times(0, M))\right) .
\end{gathered}
$$

We first obtain a uniqueness result:

Lemma 12. For arbitrary $\nu^{0} \in \mathcal{Y}_{\sharp}$ and $\mathcal{K} \Subset \mathbb{R} / L \mathbb{Z} \times(0, M)$, there exists $T_{*} \leq T$ such that (90)-(91) admits at most one solution $\nu \in C\left(\left[0, T_{*}\right] ; \mathcal{Y}_{\sharp}-w *\right)$ with support in $\mathcal{K}$.

Proof. We provide a proof with a duality-regularization argument. By difference, we prove that if $\nu$ satisfies :

- $\nu$ is a continuous function on $\left[0, T_{*}\right]$ with values in periodic measures on $\mathbb{R} \times[0, M]$ (endowed with the $w *$-topology)

- $\nu_{t}$ has support in $\mathcal{K} \Subset \mathbb{R} / L \mathbb{Z} \times(0, M)$ for arbitrary $t \in(0, T)$ and $\nu_{\mid t=0}=0$

- for arbitrary $\varphi \in \mathcal{D}((0, T) \times \mathbb{R} \times(0, M))$ we have:

$$
\int_{0}^{T}\left\langle\nu, \partial_{t} \varphi+u \cdot \nabla \varphi-g \varphi\right\rangle=0
$$

then $\nu$ vanishes globally on $[0, T]$.

First, by a standard regularization argument, we have that, for arbitrary $t \in[0, T]$ and $\varphi \in W^{1,1}\left([0, t] ; C_{c}^{1}(\mathbb{R} \times(0, M))\right)$ there holds:

$$
\left\langle\nu_{t}, \varphi(t, \cdot)\right\rangle=-\int_{0}^{t}\left\langle\nu_{s}, \partial_{t} \varphi+u \cdot \nabla \varphi-g \varphi\right\rangle \mathrm{d} s .
$$

We also fix $\mathcal{K}^{\prime}$ containing strictly $\mathcal{K}$ with $\mathcal{K}^{\prime} \Subset(\mathbb{R} / L \mathbb{Z}) \times(0, M)$ and remark that there exists $T_{*}$ depending on $u$ and $\mathcal{K}^{\prime}$ for which any characteristics $\Gamma$ of the flow associated to $u$ crossing $\mathcal{K}^{\prime}$ on $\left[0, T_{*}\right]$ satisfies $\Gamma \Subset(\mathbb{R} / L \mathbb{Z}) \times(0, M)$.

Then, we introduce mollified velocities and source term $\left(u^{\varepsilon}, g^{\varepsilon}\right)_{\varepsilon>0}$ obtained by convolution with tensorized mollifiers $\left(\rho_{\varepsilon}\right)_{\varepsilon>0}$. Given the assumed regularity on $u$ and $g$ we have that $\left(u^{\varepsilon}, g^{\varepsilon}\right) \in L^{1}\left(0, T ; C^{1}((\mathbb{R} / L \mathbb{Z}) \times[0, M])\right)$. We shall use the following convergence afterwards:

- we have the classical convergences

$$
\left\|u_{2}^{\varepsilon}-u_{2}\right\|_{L^{1}\left(0, T ; L^{\infty}\right)}+\left\|g^{\varepsilon}-g\right\|_{L^{1}\left(0, T ; L^{\infty}\right)}=0 .
$$

- thanks to (92)-(93) we have $\nabla u_{1} \in L^{1}\left(0, T ; L^{\infty}(\mathbb{R} / L \mathbb{Z} \times(0, M))\right)$ and:

$$
\left\|u_{1}^{\varepsilon}-u_{1}\right\|_{L^{1}\left(0, T ; L^{\infty}\right)} \leq C \varepsilon
$$

- applying (94)-(96) in the computations of $\partial_{2} g^{\varepsilon}$ and $\partial_{2} u_{2}^{\varepsilon}$ and $\nabla u_{1}$ we obtain the uniform bounds:

$$
\left\|\partial_{2} u_{2}^{\varepsilon}\right\|_{L^{1}\left(0, T ; L^{\infty}\right)}+\left\|\partial_{2} g^{\varepsilon}\right\|_{L^{1}\left(0, T ; L^{\infty}\right)}+\left\|\nabla u_{1}^{\varepsilon}\right\|_{L^{1}\left(0, T ; L^{\infty}\right)} \leq C .
$$


- applying (94)-(96) in the computations of $\partial_{1} g^{\varepsilon}$ and $\partial_{1} u_{2}^{\varepsilon}$ we obtain the divergences:

$$
\left\|\partial_{1} u_{2}^{\varepsilon}\right\|_{L^{1}\left(0, T ; L^{\infty}\right)}+\left\|\partial_{1} g^{\varepsilon}\right\|_{L^{1}\left(0, T ; L^{\infty}\right)} \leq \frac{C}{\sqrt{\varepsilon}} .
$$

Let now $t \in\left(0, T_{*}\right)$ and $\varphi^{\sharp} \in C_{c}^{1}(\mathbb{R} \times(0, M))$ with support in $\mathcal{K}^{\prime}$ we construct now $\varphi^{\varepsilon}$ solution to

$$
\begin{aligned}
\partial_{t} \varphi^{\varepsilon}+u^{\varepsilon} \cdot \nabla \varphi^{\varepsilon} & =g^{\varepsilon} \varphi^{\varepsilon} & \text { on }(0, t) \times \mathbb{R} \times(0, M), \\
\varphi(t, \cdot) & =\varphi^{\sharp} & \text { on } \mathbb{R} \times(0, M) .
\end{aligned}
$$

Classical results on convection equations yield that $\varphi^{\varepsilon}$ has the requested regularity to be a test-function in (98) for $\varepsilon$ sufficiently small. In particular, the convergence of the flow associated with $u^{\varepsilon}$ towards the flow associated with $u$ ensures that, for $\varepsilon$ sufficiently small, $\varphi^{\varepsilon}(s, \cdot)$ has compact support in $\mathbb{R} \times(0, M)$ for any $s \in[0, t]$. Consequently, we have

$$
\left\langle\nu_{t}, \varphi^{\sharp}\right\rangle=-\int_{0}^{t}\left\langle\nu,\left(u-u^{\varepsilon}\right) \cdot \nabla \varphi^{\varepsilon}-\left(g-g^{\varepsilon}\right) \varphi^{\varepsilon}\right\rangle \mathrm{d} s .
$$

This entails that:

$$
\left|\left\langle\nu_{t}, \varphi^{\sharp}\right\rangle\right| \leq C\left[I_{1}+I_{2}+I_{3}\right]
$$

where :

$$
\begin{aligned}
& I_{1}=\int_{0}^{t}\left\|\left(u_{1}-u_{1}^{\varepsilon}\right) \partial_{1} \varphi^{\varepsilon}\right\|_{L^{\infty}(\mathbb{R} \times(0, M))} \\
& I_{2}=\int_{0}^{t}\left\|\left(u_{2}-u_{2}^{\varepsilon}\right) \partial_{2} \varphi^{\varepsilon}\right\|_{L^{\infty}(\mathbb{R} \times(0, M))} \\
& I_{3}=\int_{0}^{t}\left\|\left(g-g^{\varepsilon}\right) \varphi^{\varepsilon}\right\|_{L^{\infty}(\mathbb{R} \times(0, M))}
\end{aligned}
$$

Concerning $I_{3}$ at first, we apply classical maximum-principle arguments yielding that, for any $s \in(0, t)$ :

$$
\left\|\varphi^{\varepsilon}(s, \cdot)\right\|_{L^{\infty}} \leq\left\|\varphi^{\sharp}\right\|_{L^{\infty}} \exp \left(\int_{0}^{t}\left\|g^{\varepsilon}\right\|_{L^{\infty}}\right) .
$$

Due to the convergence of $g^{\varepsilon}$ towards $g$ we obtain that $\varphi^{\varepsilon}$ is uniformly bounded independently of $\varepsilon$ and that

$$
\left|I_{3}\right| \leq C \int_{0}^{t}\left\|g-g^{\varepsilon}\right\|_{L^{\infty}} \rightarrow 0 \quad \text { when } \varepsilon \rightarrow 0 .
$$

Then, we differentiate the transport equation for $\varphi^{\varepsilon}$ w.r.t. $x_{2}$. As $\partial_{2} u_{1}^{\varepsilon}=0$, we obtain that $\varphi_{2}^{\varepsilon}=\partial_{2} \varphi^{\varepsilon}$ satisfies:

$$
\begin{aligned}
\partial_{t} \varphi_{2}^{\varepsilon}+u^{\varepsilon} \cdot \nabla \varphi_{2}^{\varepsilon} & =\partial_{2} g^{\varepsilon} \varphi^{\varepsilon}+g^{\varepsilon} \varphi_{2}^{\varepsilon}-\partial_{2} u_{2}^{\varepsilon} \varphi_{2}^{\varepsilon} & \text { on }(0, t) \times \mathbb{R} \times(0, M), \\
\varphi_{2}^{\varepsilon}(t, \cdot) & =\partial_{2} \varphi^{\sharp} & \text { on } \mathbb{R} \times(0, M) .
\end{aligned}
$$


Refering again to a maximum principle argument for transport equations, we obtain that, for any $s \in(0, t)$ :

$$
\left\|\varphi_{2}^{\varepsilon}(s, \cdot)\right\|_{L^{\infty}} \leq\left(\left\|\partial_{2} \varphi^{\sharp}\right\|_{L^{\infty}}+\int_{0}^{t}\left\|\partial_{2} g^{\varepsilon} \varphi^{\varepsilon}\right\|_{L^{\infty}}\right) \exp \left(\int_{0}^{t}\left\|g^{\varepsilon}\right\|_{L^{\infty}}+\left\|\partial_{2} u_{2}\right\|_{L^{\infty}}\right) .
$$

Applying the uniform bound on $\varphi^{\varepsilon}$ together with (101) we get:

$$
\left\|\varphi_{2}^{\varepsilon}(s, \cdot)\right\|_{L^{\infty}} \leq C, \quad \forall s \in(0, t) .
$$

Combining this remark with the convergence (99) we obtain then:

$$
\begin{aligned}
\left|I_{2}\right| & \leq C \sup _{(0, t)}\left\|\varphi_{2}^{\varepsilon}\right\|_{L^{\infty}} \int_{0}^{t}\left\|\left(u_{2}^{\varepsilon}-u_{2}\right)\right\|_{L^{\infty}} \\
& \leq C \int_{0}^{t}\left\|\left(u_{2}^{\varepsilon}-u_{2}\right)\right\|_{L^{\infty}} \rightarrow 0 \quad \text { when } \varepsilon \rightarrow 0 .
\end{aligned}
$$

Finally, to compute $I_{1}$ we differentiate the transport equation for $\varphi^{\varepsilon}$ w.r.t. $x_{1}$. We obtain that $\varphi_{1}^{\varepsilon}=\partial_{1} \varphi^{\varepsilon}$ satisfies:

$$
\begin{aligned}
\partial_{t} \varphi_{1}^{\varepsilon}+u^{\varepsilon} \cdot \nabla \varphi_{1}^{\varepsilon} & =\partial_{1} g^{\varepsilon} \varphi^{\varepsilon}+g^{\varepsilon} \varphi_{1}^{\varepsilon}-\partial_{1} u_{2}^{\varepsilon} \varphi_{2}^{\varepsilon}-\partial_{1} u_{1}^{\varepsilon} \varphi_{1}^{\varepsilon} & \text { on }(0, t) \times \mathbb{R} \times(0, M), \\
\varphi_{1}^{\varepsilon}(t, \cdot) & =\partial_{1} \varphi^{\sharp} & \text { on } \mathbb{R} \times(0, M) .
\end{aligned}
$$

Again, this yields that, for any $s \in(0, t)$ :

$$
\begin{aligned}
&\left\|\varphi_{1}^{\varepsilon}(s, \cdot)\right\|_{L^{\infty}} \leq\left(\left\|\partial_{1} \varphi^{\sharp}\right\|_{L^{\infty}}+\int_{0}^{t}\left(\left\|\partial_{1} g^{\varepsilon} \varphi^{\varepsilon}\right\|_{L^{\infty}}+\left\|\partial_{1} u_{2}^{\varepsilon} \varphi_{2}^{\varepsilon}\right\|_{; L^{\infty}}\right)\right) \\
& \exp \left(\int_{0}^{t}\left\|g^{\varepsilon}\right\|_{L^{\infty}}+\left\|\partial_{1} u_{1}^{\varepsilon}\right\|_{L^{\infty}}\right)
\end{aligned}
$$

Applying the uniform bound on $\varphi^{\varepsilon}$ and $\varphi_{2}^{\varepsilon}$ with (101) and (102) we conclude that

$$
\sup _{s \in(0, t)}\left\|\varphi_{1}^{\varepsilon}(s, \cdot)\right\|_{L^{\infty}} \leq \frac{C}{\sqrt{\varepsilon}}
$$

Combining this remark with the convergence (100) we obtain then:

$$
\begin{aligned}
\left|I_{1}\right| & \leq C \sup _{(0, t)}\left\|\varphi_{1}^{\varepsilon}\right\|_{L^{\infty}(\mathbb{R} \times(0, M))} \int_{0}^{t}\left\|\left(u_{1}^{\varepsilon}-u_{1}\right)\right\|_{L^{\infty}((\mathbb{R} / L \mathbb{Z}) \times(0, M))} \\
& \leq C \sqrt{\varepsilon} \rightarrow 0 \quad \text { when } \varepsilon \rightarrow 0 .
\end{aligned}
$$

Finally, we have $\left\langle\nu_{t}, \varphi^{\sharp}\right\rangle=0$ whatever the value of $\varphi^{\sharp}$. As $\nu_{t}$ has support in $\mathcal{K}$ strictly contained in $\mathcal{K}^{\prime}$ we conclude that $\nu_{t}=0$ globally. 
We then construct solutions for initial data which are convex combinations of Dirac measures. Namely, we assume that there exists $\left(\alpha_{i}^{0}, \rho_{i}^{0}\right)_{i=1, \ldots, k} \in\left[L^{\infty}(\mathbb{R} / L \mathbb{Z})\right]^{2 k}$ satisfying:

$$
\begin{aligned}
& 0 \leq \alpha_{i}^{0}(x) \leq 1 \quad \sum_{i=0}^{k} \alpha_{i}^{0}(x)=1 \quad \text { a.e. in } \mathbb{R} / L \mathbb{Z} . \\
& \frac{4}{M} \leq \rho_{i}^{0}(x) \leq \frac{M}{4} \quad \text { a.e. in } \mathbb{R} / L \mathbb{Z} .
\end{aligned}
$$

and we consider the initial data for (90) that reads:

$$
\nu^{0}=\sum_{i=1}^{k} \alpha_{i}^{0}(x) \delta_{\xi=\rho_{i}^{0}(x)} .
$$

We show that we can construct a solution to (90) with the same structure (under the above assumptions on the velocity $u$ and $g$ ). Namely, there holds:

Lemma 13. Let (103)-(104)-(105) hold true. There exists $T_{0}<T$ and

$$
\left(\alpha_{i}, \rho_{i}\right) \in L^{\infty}\left(\left(0, T_{0}\right) \times(\mathbb{R} / L \mathbb{Z})\right) \cap C\left(\left[0, T_{0}\right] ; L^{1}(\mathbb{R} / L \mathbb{Z})\right)
$$

satisfying

$$
\begin{aligned}
& 0 \leq \alpha_{i}(t, x) \leq 1 \quad \sum_{i=0}^{k} \alpha_{i}(t, x)=1 \quad \text { a.e. } \\
& \frac{2}{M} \leq \rho_{i}(t, x) \leq \frac{M}{2} \quad \text { a.e. }
\end{aligned}
$$

such that $\nu=\sum_{i=1}^{k} \alpha_{i} \delta_{\xi=\rho_{i}} \in C\left(\left[0, T_{0}\right] ; \mathcal{Y}_{\sharp}\right)$ is a solution to (90)-(91).

Proof. The proof is straightforward. Let $\nu=\sum_{i=1}^{k} \alpha_{i} \delta_{\xi=\rho_{i}}$ with $\left(\alpha_{i}, \rho_{i}\right)$ as in the statement of the theorem. We have thus that, for arbitrary $\phi \in C_{c}(\mathbb{R} \times[0, M])$, there holds:

$$
\left\langle\nu_{t}, \phi\right\rangle=\int_{0}^{L} \sum_{i=1}^{k} \alpha_{i}(t, x) \phi\left(x, \rho_{i}(t, x)\right) \mathrm{d} x .
$$

Hence $\langle\nu, \phi\rangle \in C\left(\left[0, T_{0}\right]\right)$ with $\left|\left\langle\nu_{t}, \phi\right\rangle\right| \leq L\|\phi\|_{L_{\sharp}^{\infty}}$ so that we have indeed $\nu \in C\left(\left[0, T_{0}\right] ; \mathcal{Y}_{\sharp}\right)$.

Then, applying a classical density argument, we obtain that $\nu$ is charaterized by its action on tensorized test-functions $(x, \xi) \mapsto \psi(x) \beta(\xi)$. Plugging $\psi \otimes \beta$ as test-function in (90)-(91), we obtain the following equations:

$$
\begin{aligned}
\partial_{t} \sum_{i=1}^{k} \alpha_{i} \beta\left(\rho_{i}\right)+\partial_{1} \sum_{i=1}^{k} \alpha_{i} \beta\left(\rho_{i}\right) u_{1}\left(\cdot, \cdot, \rho_{i}\right) & =\sum_{i=1}^{k} \alpha_{i} u_{2}\left(\cdot, \cdot, \rho_{i}\right) \beta^{\prime}\left(\rho_{i}\right)-\sum_{i=1}^{k} \alpha_{i} g\left(\cdot, \cdot, \rho_{i}\right) \beta\left(\rho_{i}\right) . \\
\sum_{i=1}^{k} \alpha_{i} \beta\left(\rho_{i}\right)_{\mid t=0} & =\sum_{i=1}^{k} \alpha_{i}^{0} \beta\left(\rho_{i}^{0}\right) .
\end{aligned}
$$


Finally, we obtain that $\nu$ is a solution to (90)-(91) if the $\left(\alpha_{i}, \rho_{i}\right)$ satisfy simultaneously:

$$
\begin{aligned}
\partial_{t} \alpha_{i}+\partial_{1}\left(\alpha_{i} u_{1}\right)+\alpha_{i} g\left(\cdot, \cdot, \rho_{i}\right) & =0 \\
{\left[\alpha_{i}\right]_{\mid t=0} } & =\alpha_{i}^{0}
\end{aligned}
$$

and

$$
\begin{aligned}
\partial_{t} \rho_{i}+u_{1} \partial_{1} \rho_{i}-u_{2}\left(\cdot, \cdot, \rho_{i}\right) & =0 \\
{\left[\rho_{i}\right]_{\mid t=0} } & =\rho_{i}^{0} .
\end{aligned}
$$

Remark that we introduced that $u_{1}$ does not depend on $\rho_{i}$ (by assumption). Existence of a solution

$$
\left(\alpha_{i}, \rho_{i}\right) \in L^{\infty}\left(\left(0, T_{0}\right) \times(\mathbb{R} / L \mathbb{Z})\right) \cap C\left(\left[0, T_{0}\right] ; L^{1}(\mathbb{R} / L \mathbb{Z})\right)
$$

to this system satisfying (106)-(107) follows from a straightforward adaptation of Di PernaLions arguments in the spirit of [6, Lemma 2].

\section{Appendix B. Formal CAlCulation versus Young measure method}

Let us compare in this appendix the system obtained through a formal WKB method and the system derived using kinetic formulation and characterization of the Young measures. With the Young measure method in the two-fluid setting, we get the following equation on $\alpha_{+}$:

$$
\partial_{t} \alpha_{+}+u \partial_{x} \alpha_{+}+\alpha_{+} \partial_{x} u=\frac{\alpha_{+}}{\mu_{+}}\left[\frac{1}{\frac{\alpha_{+}}{\mu_{+}}+\frac{\alpha_{-}}{\mu_{-}}}\left(\partial_{x} u-\left(\alpha_{+} \frac{p_{+}}{\mu_{+}}+\alpha_{-} \frac{p_{-}}{\mu_{-}}\right)\right)+p_{+}\right]
$$

Thus we get the following equation

$$
\partial_{t} \alpha_{+}+u \partial_{x} \alpha_{+}=\kappa_{1} \partial_{x} u+\kappa_{2}
$$

with

$$
\begin{aligned}
\kappa_{1} & =\frac{\alpha_{+}}{\mu_{+}} \frac{1}{\frac{\alpha_{+}}{\mu_{+}}+\frac{\alpha_{-}}{\mu_{-}}}-\alpha_{+} \\
& =\frac{\alpha_{+}}{\mu_{+}} \frac{1-\mu_{+}\left(\frac{\alpha_{+}}{\mu_{+}}+\frac{\alpha_{-}}{\mu_{-}}\right)}{\frac{\alpha_{+}}{\mu_{+}}+\frac{\alpha_{-}}{\mu_{-}}} \\
& =\frac{\alpha_{+} \alpha_{-}}{\mu_{+}} \frac{1-\frac{\mu_{+}}{\mu_{-}}}{\frac{\alpha_{+}}{\mu_{+}}+\frac{\alpha_{-}}{\mu_{-}}}=\frac{\alpha_{+} \alpha_{-}\left(\mu_{-}-\mu_{+}\right)}{\alpha_{+} \mu_{+}+\alpha_{-} \mu_{-}} .
\end{aligned}
$$


and

$$
\begin{aligned}
\kappa_{2} & =\frac{\alpha_{+}}{\mu_{+}}\left[p_{+}-\frac{1}{\frac{\alpha_{+}}{\mu_{+}}+\frac{\alpha_{-}}{\mu_{-}}}\left(\alpha_{+} \frac{p_{+}}{\mu_{+}}+\alpha_{-} \frac{p_{-}}{\mu_{-}}\right)\right] \\
& =\frac{\alpha_{+} \alpha_{-}}{\mu_{+} \mu_{-}} \frac{1}{\frac{\alpha_{+}}{\mu_{+}}+\frac{\alpha_{-}}{\mu_{-}}}\left(p_{+}-p_{-}\right) . \\
& =\frac{\alpha_{+} \alpha_{-}}{\alpha_{-} \mu_{+}+\alpha_{+} \mu_{-}}\left(p_{+}-p_{-}\right) .
\end{aligned}
$$

This reads

$$
\partial_{t} \alpha_{+}+u \partial_{x} \alpha_{+}=\frac{\alpha_{+} \alpha_{-}}{\alpha_{-} \mu_{+}+\alpha_{+} \mu_{-}}\left[\left(p_{+}-p_{-}\right)+\left(\mu_{-}-\mu_{+}\right) \partial_{x} u\right] .
$$

As for the momentum equation, we obtain :

$$
\partial_{t}(\rho u)+\partial_{x}\left(\rho u^{2}\right)-\partial_{x}\left(m^{\infty} \partial_{x} u\right)+\partial_{x} \pi^{\infty}=0,
$$

where

and $\pi^{\infty}=m^{\infty} \kappa^{\infty}$ with

$$
m^{\infty}=\frac{1}{\frac{\alpha_{+}}{\mu_{+}}+\frac{\alpha_{-}}{\mu_{-}}}=\frac{\mu_{+} \mu_{-}}{\alpha_{+} \mu_{-}+\alpha_{-} \mu_{+}}
$$

and thus :

$$
\kappa^{\infty}=\alpha_{+} \frac{p_{+}}{\mu_{+}}+\alpha_{-} \frac{p_{-}}{\mu_{-}}
$$

$$
\pi^{\infty}=m^{\infty} \kappa^{\infty}=\frac{\alpha_{+} p_{+} \mu_{-}+\alpha_{-} p_{-} \mu_{+}}{\alpha_{+} \mu_{-}+\alpha_{-} \mu_{+}} .
$$

This is, up to the notations, the system obtained using the WKB method.

Acknowledgements. The authors are partially supported by the ANR-13-BS01-0003-01 project DYFICOLTI.

\section{REFERENCES}

[1] A.A. Amosov, A.A. Zlotnik. On the error of quasi-averaging of the equations of motion of a viscous barotropic medium with rapidly oscillating data. Comp. Maths. Math. Phys, 36, 10, 14151428, (1996).

[2] D. Bresch. Topics on compressible Navier-Stokes equations with non degenerate viscosities. contributions by R. Danchin, A. Novotny, M. Perepetlisa. Panorama et synthèses, submitted (2014).

[3] D. Bresch. Shallow-water equations and related topics. Handbook of Differential Equations, Evolutionary equations, vol. 5, Edited by C.M. Dafermos and M. Pokorny, (2009), 1-102.

[4] D. Bresch, B. Desjardins. On the existence of global weak solutions to the Navier-Stokes equations for viscous compressible and heat conducting fluids. J. Math. Pures et Appl., 57-90 (2007).

[5] D. Bresch, B. Desjardins. Sur la théorie globale des équations de Navier-Stokes compressibles, Actes des 33èmes journées "Equations aux dérivées Partielles, Evian (2006).

[6] D. Bresch, M. Hillairet. Note on the derivation of multicomponent flow systems. Proc. AMS, $143,3429-3443,(2015)$.

[7] D. Bresch, X. Huang. A multi-fluid compressible system as the limit of weak solutions of the isentropic compressible Navier-Stokes equations. Arch. Ration. Mech. Anal., 201(2):647-680, (2011). 
[8] D. Drew, S.L. Passman. Theory of multicomponent fluids. Applied Math Sciences, (1999).

[9] E. Feireisl, A. Novotny, H. Petzeltova. On the existence of globally defined weak solutions to the Navier-Stokes equations. J. Math. Fluid Mech., 3, 358-392, (2001).

[10] B. HASPOT. Existence of global strong solution for the compressible Navier-Stokes equations with degenerate viscosity coefficients in 1D. See arXiv:1411.5503

[11] Y. Kanel. On a model system of equations of one-dimensional gas motion, Diff. Eq. 4, 374-380, (1968).

[12] W. E. Propagation of oscillations in the solutions of 1-D compressible fluid equations. Comm. Partial Differential Equations, 17(3-4):347-370, (1992).

[13] M. Hillairet. Propagation of density-oscillations in solutions to barotropic compressible NavierStokes system. J. of Math. Fluid Mech. (9), 343-376, (2007).

[14] M. Ishi, T. HiBiki. Thermo-fluid dynamics of two-phase flow. Springer (2006).

[15] P.-L. Lions. Mathematical topics in fluid mechanics, Vol. II: compressible models. Oxford Lect. Ser. Math. Appl. (1998).

[16] A. Mellet, A. Vasseur. Existence and Uniqueness of global strong solutions for one-dimensional compressible Navier-Stokes equations. SIAM J. Math. Anal. 39 (2007/08), no. 4, 1344-1365.

[17] P. Plotnikov, J. Sokolowski. Compressible Navier-Stokes equations, Theory and Shape Optimization. Series: Monografie Matematyczne. Birkhäuser Basel (2012).

[18] D. SERRE. Variations de grande amplitude pour la densité dun fluide visqueux compressible. Phys. $D, 48(1): 113-128,(1991)$.

[19] D. Serre. Asymptotics of homogeneous oscillations in a compressible viscous fluid. Bul. Soc. Bras. Mat, 32, 535-442 (2001).

$[20] \mathrm{V}$. A. Solonnikov. The solvability of the initial-boundary value problem for the equations of motion of a viscous compressible fluid. Zap. Naucn. Sem. Leningrad. Otdel. Mat. Inst. Steklov. (LOMI), 56: 128-142, 197, 1976. Investigations on linear operators and theory of functions, VI.

[21] L. TARTAR. The compensated compactness method applied to systems of conservation laws. In Systems of nonlinear partial differential equations (Oxford, 1982), volume 111 of NATO Adv. Sci. Inst. Ser. C Math. Phys. Sci., pages 263-285. Reidel, Dordrecht, 1983. 